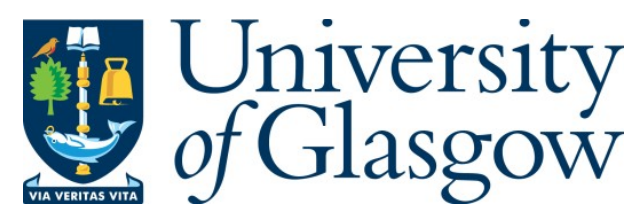

Viale, A. , Ceriotti, M. and McInnes, C. (2020) Dynamics of an orbital siphon anchored to a rotating ellipsoidal asteroid for resource exploitation. Acta Astronautica, 177, pp. 561-576.

(doi: 10.1016/j.actaastro.2020.08.001)

This is the Author Accepted Manuscript.

There may be differences between this version and the published version. You are advised to consult the publisher's version if you wish to cite from it.

$\underline{\text { https://eprints.gla.ac.uk/221657/ }}$

Deposited on: 3 August 2020

Enlighten - Research publications by members of the University of Glasgow http://eprints.gla.ac.uk 


\title{
Dynamics of an orbital siphon anchored to a rotating ellipsoidal asteroid for resource exploitation
}

\author{
Andrea Viale ${ }^{1, *}$, Matteo Ceriotti ${ }^{2, *}$, Colin McInnes ${ }^{3, *}$ \\ University of Glasgow, Glasgow, Scotland G12 8QQ, United Kingdom
}

\begin{abstract}
This paper investigates the dynamics of an orbital siphon anchored to a rotating ellipsoidal asteroid. The siphon is a chain of tether-connected payload masses arranged vertically from the asteroid surface, envisaged for propellantless delivery of payloads (e.g., mined material) from the asteroid surface to a collecting spacecraft. If the structure is long enough, the centrifugal-induced force can overcome the gravitational force on the payloads, eventually allowing resource payloads to escape. By connecting new payloads at the bottom of this chain while removing upper payloads a net orbital siphon effect is established, which provides a net continuous flow of resources from the asteroid surface to a collecting spacecraft, attached at the top of the siphon. The dynamics of the siphon is investigated in detail by varying a set of relevant parameters, in particular, chain length, anchor location and asteroid shape. It is shown that the system exhibits oscillatory behaviour in the equatorial plane, with decreasing oscillation amplitude over time and that the longest equatorial end is the best anchor location to guarantee proper siphon operation while minimizing the chain length. Eventually, a method is proposed to exploit the equatorial Coriolis-induced oscillations of the siphon to transfer payload masses from the collecting spacecraft to the stable equilibrium points associated with the effective potential of the ellipsoidal asteroid, where a catcher would collect the material.
\end{abstract}

Keywords: asteroid engineering, orbital siphon, space resources

\footnotetext{
${ }^{*}$ Corresponding author

${ }^{1}$ Ph.D. Candidate. Department of Systems, Power and Energy. James Watt School of Engineering; a.viale.1@research.gla.ac.uk

${ }^{2}$ Lecturer in Space Systems Engineering. Department of System, Power and Energy. James Watt School of Engineering; matteo.ceriotti@glasgow.ac.uk

${ }^{3}$ Professor of Engineering Science. Department of Systems, Power and Energy. James Watt School of Engineering; colin.mcinnes@glasgow.ac.uk
} 


\section{Introduction}

Recent Earth-based observations and robotic missions have shown that some near-Earth asteroids are abundant in useful resources such as metals (in particular platinum-group metals) and water [1. Exploitation of such resources could revolutionize the future of space exploration [2, 1]. Water, for example, could provide consumables and propellant, whereas metals can be exploited for in-situ manufacturing, for example, to assemble space structures.

Research has focused on the design of asteroid mining missions, investigating models to understand costs and mission design [3, 4. However, the problem of gathering material from the surface of an asteroid for delivery to an orbiting collecting/processing spacecraft is still largely unexplored. Some authors proposed the direct launch of material into orbit (to a collecting spacecraft) [5] or for material sorting (using solar radiation pressure) [6]. However, both strategies are affected by uncertainties when launching material from the asteroid surface, for example for repeatability of mass driver launches.

An alternative method has been proposed [7, which exploits the natural rotational self-energy of the asteroid to overcome the low gravity at the surface and raise resource payloads without the need for external work to be done. The idea has its root in the space elevator concept, firstly developed by Tsiolkovski [8, then improved by Artustanov and Pearson [9]. The elevator, initially conceived as a thought experiment for Earth applications, is envisaged as a tethered structure, where the tether is kept in equilibrium by the balance of centrifugal-induced and gravitational forces acting on it: a payload can then by raised to a desired altitude and, once synchronous orbit is reached, the payload can increase its altitude without any further work required.

A direct evolution of the space elevator is the orbital siphon concept, firstly introduced by Davis and elaborated by McInnes [10]. The orbital siphon is a tether-connected chain payloads, comprising material from the asteroid surface: if the structure is long enough, the centrifugal-induced force can overcome the gravitational force on the payloads, eventually allowing resource payloads to escape. If new payloads are then connected at the bottom of this chain of masses while the upper payloads are removed, an orbital siphon effect can be envisaged. This provides a continuous flow of resources from the surface of the asteroid to bound orbit or escape (depending on the siphon length), without the need for external work to be done.

Fast-rotating asteroids represent a suitable target for the orbital siphon, as the typical ratio between the centrifugal-induced force to gravitational force at their equator can be close to unity, 
thus reducing the chain length required for the orbital siphon effect to take place. Viale et al. [7] analysed in detail the dynamics of an orbital siphon anchored to a spherical asteroid, taking into account the effects of material removal from the asteroid. In particular, it was shown that, if the material removal process continues in the long term, the rotational period of the asteroid increases to conserve the overall angular momentum of the system. This constrains the maximum amount of mass which can be removed from the asteroid. For a constant-length siphon and an asteroid spinning at its critical period, this limit was found to be approximately $12 \%$ the initial mass of the asteroid [7.

In Ref. [1] oscillations of the system in the equatorial plane were analysed. Moreover, the use of a ballast was envisaged to provide tension and keep the oscillations small. In Ref. [12, shape models of two candidate near-Earth asteroids were used to model the gravitational potential and the siphon designed as a self-supporting structure. Moreover, the behaviour of a siphon with a moving base was considered.

In this paper, the asteroid is modelled as a triaxial ellipsoid. This choice allows an estimation of the siphon behaviour as a function of the asteroid shape, by changing the asteroid semi-major axes. Key parameters, such as siphon oscillation amplitude, velocity of the chain and tether torques are taken into account, considering the effect of a collecting spacecraft receiving material at the top of the siphon. Furthermore, it is proposed to exploit the stable equilibrium points associated with the effective gravitational potential of the asteroid as depots for material storage. A method is presented to exploit the Coriolis-induced oscillation of the siphon to insert payload masses into trajectories intersecting the stable equilibrium points, where a catcher would collect the material for storing or later processing.

The paper is divided in two parts. In the first part the dynamics of the oscillating orbital siphon is discussed and the second part is a preliminary analysis of material transfer to the equilibrium points exploiting the siphon dynamics.

\section{Asteroid model}

The asteroid is modelled as a triaxial ellipsoid with semi-major axes $\bar{\alpha} \geq \bar{\beta} \geq \bar{\gamma}$ and constant density $\rho_{A}$, rotating with constant angular velocity $\bar{\omega}$ about the axis with largest inertia. A corotating reference frame is defined such that the $x$-axis lies along the largest dimension $\bar{\alpha}$, the $y$-axis lies along the intermediate dimension $\bar{\beta}$ and the $z$-axis lies along the smallest dimension $\bar{\gamma}$, parallel 
to the angular velocity vector. The gravitational parameter $\mu$ of the asteroid is therefore computed as

$$
\mu=\frac{4}{3} \pi G \rho_{A} \bar{\alpha} \bar{\beta} \bar{\gamma}
$$

where $G=6.67 \times 10^{-11} \mathrm{~m}^{3} \mathrm{~kg}^{-1} \mathrm{~s}^{-2}$ is the gravitational constant.

To provide clarity and generality, non-dimensional variables will be used. In particular, distance variables are scaled by $\bar{\alpha}$, time variables are scaled by $\bar{\omega}^{-1}$ and mass variables are scaled by the mass $m_{l s}$ of an element of the lifting side of the siphon, which will be defined later in Sect. 3 . All derived units can be obtained from these scale factors (unless explicitly specified in the text).

At any point outside the asteroid an effective potential $U$ can be defined, which combines the effects of the centrifugal-induced acceleration due to the asteroid rotation and the gravitational acceleration. In particular:

$$
U=\frac{1}{2}\left(x^{2}+y^{2}+z^{2}\right)-\frac{1}{\omega^{2}} V
$$

where the first term represents the centrifugal potential while $V$ is the gravitational potential, with

$$
\omega=\frac{\bar{\omega}}{\sqrt{\frac{4 G \rho_{A} \pi \beta \gamma}{3}}}=\frac{\bar{\omega}}{\sqrt{\mu / \bar{\alpha}^{3}}}
$$

The parameter $\bar{\omega}$ is effectively the square root of the ratio between the centripetal acceleration and the gravitational acceleration acting on a particle at the longest end of the ellipsoid, assuming that the entire mass of the asteroid is concentrated at the origin. If the asteroid is a sphere, $\omega^{2}$ represents the true ratio between the centrifugal-induced acceleration and the gravitational acceleration at its surface.

From MacMillan [13], the gravitational potential $V$ used in Eq. (2) can be expressed analytically in non-dimensional form as:

$$
V=\frac{3}{4} \int_{\lambda}^{\infty} \phi(x, y, z, \nu) \frac{d \nu}{\Delta(\nu)}
$$

with

$$
\begin{aligned}
\Delta(\nu) & =\sqrt{(1+\nu)\left(\beta^{2}+\nu\right)\left(\gamma^{2}+\nu\right)} \\
\phi(x, y, z, \nu) & =\frac{x^{2}}{1+\nu}+\frac{y^{2}}{\beta^{2}+\nu}+\frac{z^{2}}{\gamma^{2}+\nu}-1 .
\end{aligned}
$$


The parameter $\lambda>0$, which is a function of $(x, y, z)$, is obtained from the condition

$$
\phi(x, y, z, \lambda)=0 .
$$

Equation 7 defines the ellipsoid passing through the point $(x, y, z)$ which is confocal to the body's ellipsoid and it has a unique positive root whenever $\phi(x, y, z, 0)>0$. (i.e., the point lies outside the ellipsoid, as in the present case). For a point on the asteroid surface $\lambda=0$. The interested reader should refer to [13] for an exhaustive derivation of the potential.

Note that, by using non-dimensional coordinates, the effective potential at any point at the asteroid is completely defined by the three parameters $\beta, \gamma$ and $\omega$. If the density is fixed, $\beta, \gamma$ and $\bar{\omega}$ (or the asteroid period $2 \pi / \bar{\omega}$ ) are sufficient to describe its behaviour. Since it is easier to consider period variation rather than $\omega$ (note that $\omega$ depends both on the period and the asteroid shape, as dictated by Eq. (3)), a constant asteroid density of $2.5 \mathrm{~g} / \mathrm{cm}^{3}$ is considered in in Sects. 2 , 3 and 4 . When necessary, comments related to larger/lower asteroid densities will be made.

\subsection{Equations of motion and equilibrium points}

The differential equation governing the motion of a point mass in the rotating reference frame can be written as

$$
\begin{aligned}
\ddot{x}-2 \dot{y} & =U_{x} \\
\ddot{y}+2 \dot{x} & =U_{y} \\
\ddot{z} & =U_{z}
\end{aligned}
$$

where $U_{x}, U_{y}, U_{z}$ are the partial derivatives of the effective potential with respect to the variables $x, y$ and $z$ respectively:

$$
\begin{aligned}
& U_{x}=x\left[1-\frac{3}{2 \omega^{2}} \int_{\lambda}^{\infty} \frac{\phi_{x} d \nu}{(1+\nu) \Delta(\nu)}\right] \\
& U_{y}=y\left[1-\frac{3}{2 \omega^{2}} \int_{\lambda}^{\infty} \frac{\phi_{y} d \nu}{\left(\beta^{2}+\nu\right) \Delta(\nu)}\right] \\
& U_{z}=z\left[1-\frac{3}{2 \omega^{2}} \int_{\lambda}^{\infty} \frac{\phi_{z} d \nu}{\left(\gamma^{2}+\nu\right) \Delta(\nu)}\right] .
\end{aligned}
$$




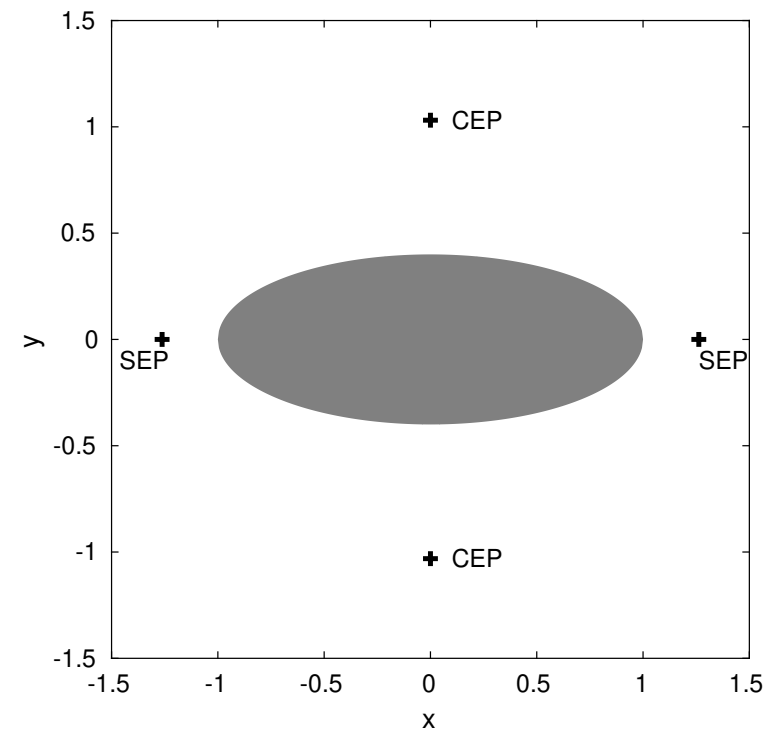

Figure 1: Location of the equilibrium points for an asteroid with $\beta=\gamma=0.4$ and period $6 \mathrm{~h}$.

By studying the condition $\nabla U=0$, four equilibrium points (EPs) can be found: two saddle equilibrium points (SEPs) on the $x$-axis and two centre points (CEPs) on the $y$-axis, both pairs being symmetrical with respect to the origin. In these positions the net force acting on a particle will vanish. Figure 1 shows the location of the four EPs for an asteroid with $\beta=\gamma=0.4$ and a rotational period of $6 \mathrm{~h}$.

It can be shown that the SEPs are always unstable, whereas CEP stability depends on the asteroid shape parameters $\beta, \gamma$ and its angular velocity $\omega$. The reader is referred to Ref. 14 for a thorough analysis on stability. Figure 2 shows the region of stability of the CEPs as a function of $\beta$ and $\omega$, assuming $\gamma=\beta$ (continuous line) and $\gamma=\beta / 2$ (dotted line), following the analysis in Ref. 14]. For a given set of shape parameters, the CEPs are unstable beneath the associated curve. The two dotted lines represent the case where the CEPs fall below the asteroid surface (the dotted black line refers to the case $\gamma=\beta$, whereas the dotted red line refers to $\gamma=\beta / 2$ ). It is apparent that fast-rotators are generally characterized by unstable CEPs. For $\gamma=\beta$, CEPs are below the surface for periods between $1.75 \mathrm{~h}$ and $2.3 \mathrm{~h}$ in the range of $\beta$ shown in the figure. 


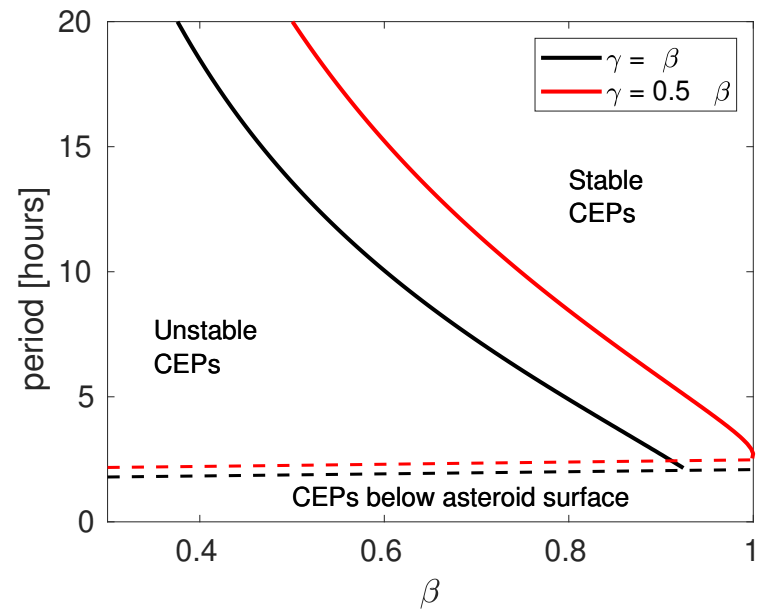

Figure 2: Regions of stability of the centre equilibria, for $\gamma=\beta$ and $\gamma=\beta / 2$, as a function of $\beta$ and the asteroid period. The dotted lines mark the region where centre points fall below the surface of the asteroid.

\section{Orbital Siphon model}

The key idea of the orbital siphon is that a chain of tether-connected payload masses arranged vertically from the surface of a rotating asteroid can be used to deliver payloads from the asteroid surface to orbit (or to a collecting spacecraft): if the chain is long enough, a net radial force is established on the chain, which would lift the chain without the need for external work to be done: new payloads are connected to the bottom of the chain while top payloads are released [7.

Here, the orbital siphon is modelled as a chain of $2 n$ masses, divided in a lifting-side (LS) and a descending-side (DS) each one with $n$ masses, as represented in Fig. 3 . The former contains bucket masses $m_{\mathrm{b}}$ filled with payload masses $m_{p}$ to be lifted, whereas the latter contains the empty buckets $m_{b}$ going back to the asteroid for refilling. Each bucket is connected to its two neighbours with a tether, and two pulleys at the two endpoints (at the anchoring point and at the top of the chain) connect the LS with the DS. The bucket chain slides without friction on a rigid rod with length $L$, which allows rotation on the equatorial plane with respect to the fixed anchor point $A$. The choice of a rigid rod is required as dynamical constraint for the model used, however it is shown in [12 that a self-sustaining siphon without support structure exhibits similar behaviour to the system described here. At the top of the rigid rod a collecting spacecraft (CS), here modelled as point mass $M$, collects the payloads released by the LS. Note that the CS mass $M$ increases over time, as more payloads are released. 
It is assumed that all the payloads have constant mass $m_{p}$. Therefore, an element on the LS will have a mass $m_{l s}=m_{b}+m_{p}$ whereas an element on the DS has mass $m_{d s}=m_{b}$. Moreover, it is assumed that the mass of the asteroid remains constant, implying that the mass removed is small (the reader is referred to [7] for a general discussion about asteroid mass variation using a simplified orbital siphon model).

Some additional assumptions are made.

1. Any friction due to the bucket-pulley interaction is neglected.

2. Other external perturbations (e.g., third-body perturbation, solar radiation pressure) are not considered.

3. The dynamics is restricted to motion in the equatorial plane $x-y$.

4. The tethers connecting consecutive buckets are assumed massless and infinitely rigid, with constant length $l=L / n$.

Although the last hypothesis may appear restrictive, it has been shown in [7] that, for realistic tether stiffness and damping parameters, the simulation results do not change significantly with respect to the case with infinitely rigid tethers. Moreover, it is reasonable to assume that the tether mass is small with respect to the masses $m_{l s}$ and $m_{d s}$. As a consequence, the entire LS and DS are effectively treated as rigid bodies sliding on a rod and the overall dynamics of the siphon can be described via two generalized coordinates: the rotation of the siphon $\theta$ and the distance of the first bucket mass of the LS from the anchor point. All rotations are assumed positive when measured from the $x$-axis in the counter-clockwise direction.

\subsection{Siphon radial force}

The coordinates of the $i$-th mass $(1 \leq i \leq 2 n)$ on the chain can now be written as

$$
\begin{aligned}
& x_{i}=r_{A} \cos \phi_{A}+s_{i} \cos \left(\phi_{A}+\theta\right) \\
& y_{i}=r_{A} \sin \phi_{A}+s_{i} \sin \left(\phi_{A}+\theta\right)
\end{aligned}
$$

where $\theta$ is the angle between the chain radial unit vector $\hat{\mathbf{u}}_{r}$ and the line $O A$ (see Fig. 3) while $r_{A}$ and $\phi_{A}$ are the polar coordinates of the anchor point, related by the ellipse equation written with 


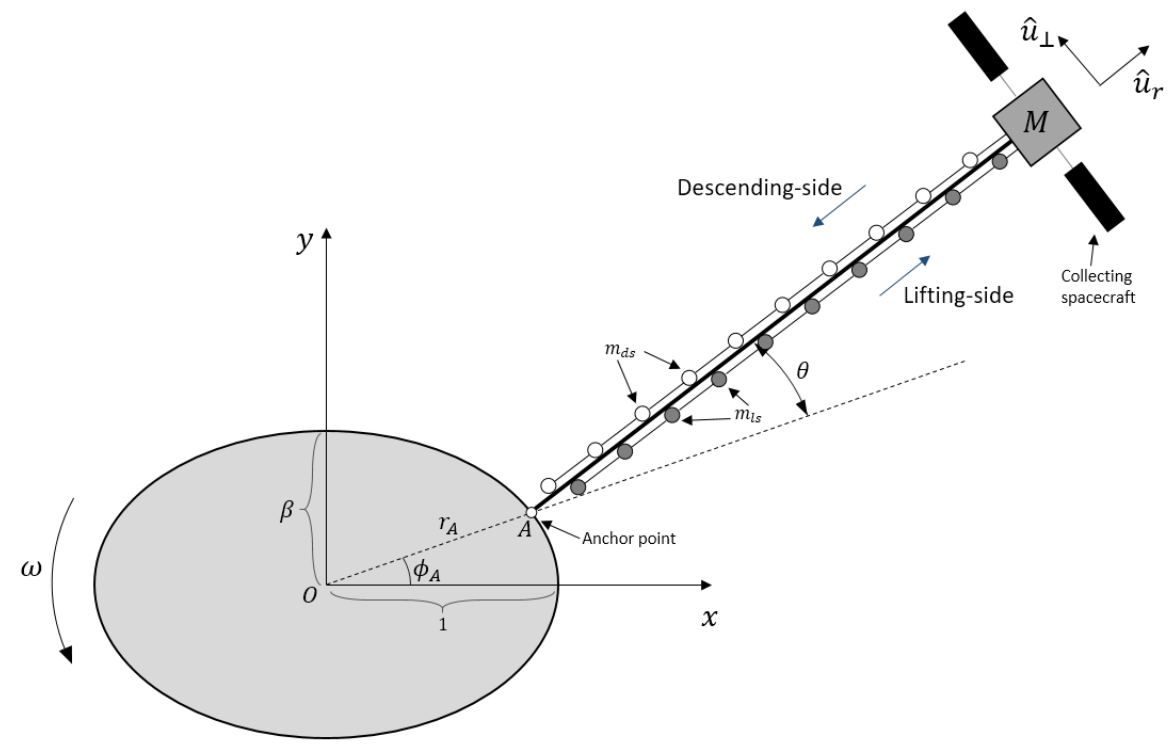

Figure 3: Orbital siphon model. Rotations are measured positive in the counter-clockwise direction.

respect to its centre:

$$
r_{A}=\frac{\beta}{\sqrt{1-\left(1-\beta^{2}\right) \cos ^{2}\left(\phi_{A}\right)}} .
$$

The parameter $s_{i}$ is the distance between the $i$-th bucket and the anchor point $A$, which can be written as a function of $s_{1}$, such that

$$
s_{i}= \begin{cases}s_{1}+(i-1) l, & 1 \leq i \leq n \\ L-\left(s_{1}+(i-1) l\right), & n+1 \leq i \leq 2 n\end{cases}
$$

The force $f_{r, i}$ acting on the $i$-th element of the chain in the radial direction $\hat{\mathbf{u}}_{r}$ is given by

$$
f_{r, i}=\left\{\begin{array}{cl}
m_{l s}\left(\nabla U\left(s_{i}\right) \cdot \hat{\mathbf{u}}_{r}+s_{i} \dot{\theta}^{2}+2 \dot{\theta} s_{i}\right), & 1 \leq i \leq n \\
-\quad m_{d s}\left(\nabla U\left(s_{i}\right) \cdot \hat{\mathbf{u}}_{r}+s_{i} \dot{\theta}^{2}+2 \dot{\theta} s_{i}\right), & n+1 \leq i \leq 2 n
\end{array}\right.
$$


The internal tension forces are not explicitly listed here as they will vanish in a summation to be performed later. Due to its significance in later analysis $m_{d s}$ is redefined as:

$$
D=m_{d s}=\frac{\bar{m}_{d s}}{\bar{m}_{l s}}=\frac{1}{1+\frac{\bar{m}_{p}}{\bar{m}_{\mathrm{b}}}}
$$

Note that $D$ can be written as a function of the ratio between the payload mass and the bucket mass and $D \in[0,1]$.

The first term of Eq. (13) is the force associated to the gradient of the effective potential $U$, defined by Eq. (2). The second and third terms are due to the chain dynamics and are non-zero if the chain is rotating with $\dot{\theta} \neq 0$. In particular, the term $s \dot{\theta}^{2}$ is the centrifugal-induced acceleration, while the last term is the Coriolis acceleration in the direction $\hat{\mathbf{u}}_{r}$, associated with the velocity $\dot{\theta} s$ in the $\hat{\mathbf{u}}_{\perp}$ direction. Note that the contribution of the terms involving the chain angular velocity $\dot{\theta}$ is negative for $-2<\dot{\theta}<0, \forall i \leq 1 \leq n$. Therefore, if the chain is rotating in the clockwise direction with angular velocities up to two times the angular velocity of the asteroid, then the chain rotation induces a net radial force contribution opposite to $\hat{\mathbf{u}}_{r}$. The unit vectors $\hat{\mathbf{u}}_{r}$ and $\hat{\mathbf{u}}_{\perp}$ can be written as a function of the anchor longitude and the chain angle as:

$$
\begin{gathered}
\hat{\mathbf{u}}_{r}=\left\{\begin{array}{c}
\cos \left(\phi_{A}+\theta\right) \\
\sin \left(\phi_{A}+\theta\right)
\end{array}\right\} \\
\hat{\mathbf{u}}_{\perp}=\left\{\begin{array}{c}
-\sin \left(\phi_{A}+\theta\right) \\
\cos \left(\phi_{A}+\theta\right)
\end{array}\right\}
\end{gathered}
$$

Then, the overall radial force acting on the chain can be written as

$$
\begin{array}{r}
f_{r}=\sum_{i=1}^{n}\left[\left(\nabla U\left(s_{i}\right) \cdot \hat{\mathbf{u}}_{r}+s_{i} \dot{\theta}^{2}+2 \dot{\theta} s_{i}\right)\right]+ \\
-\sum_{i=n+1}^{2 n}\left[D\left(\nabla U\left(s_{i}\right) \cdot \hat{\mathbf{u}}_{r}+s_{i} \dot{\theta}^{2}+2 \dot{\theta} s_{i}\right)\right] .
\end{array}
$$

When the siphon is loaded with small payload masses $m_{p} \rightarrow 0$ then $D \rightarrow 1$ (see Eq. (14)) and the the contribution of the DS on the radial force is maximum, whereas if the mass of the payload is much larger than the mass of the bucket then $D \rightarrow 0$ and the DS effect on $f_{r}$ becomes negligible. In particular, if $D=1$ and the LS and DS are aligned (this occurs when $s=l / 2$ ) then the radial 
force is zero, regardless of the other parameters. Note that in this model the CS does not influence the radial force.

\subsection{Siphon torque}

While material is moving towards the CS, Coriolis forces due to the motion of the LS (DS) will induce a clockwise (counter-clockwise) torque. The torque $T_{i}$ due to the $i$-th element of the chain with respect to the anchor point can be written as

$$
T_{i}= \begin{cases}m_{l s}\left[\nabla U\left(s_{i}\right) \cdot \hat{\mathbf{u}}_{\perp}-2(1+\dot{\theta}) \dot{s}_{1}\right] s_{i}, & 1 \leq i \leq n \\ m_{d s}\left[\nabla U\left(s_{i}\right) \cdot \hat{\mathbf{u}}_{\perp}+2(1+\dot{\theta}) \dot{s}_{1}\right] s_{i}, & n+1 \leq i \leq 2 n\end{cases}
$$

The first term is the contribution due to the gradient of the effective potential $U$ in the transversal direction $\hat{\mathbf{u}}_{\perp}$. The second part is the contribution due to the Coriolis force in the direction $\hat{\mathbf{u}}_{\perp}$ due to the motion of the chain (note that this contribution is opposite between LS and DS, since the buckets are travelling in opposite directions in the two cases).

Similarly, the torque generated by the CS with respect to the anchor point is

$$
T_{\mathrm{cs}}=M L \nabla U(L) \cdot \hat{\mathbf{u}}_{\perp}
$$

where $\nabla U(L)$ is the gradient of the effective potential at the location of the CS. Then, the overall torque $T$ of the system chain and CS is the sum $T_{c s}+\sum_{i=1}^{2 n} T_{i}$ :

$$
\begin{aligned}
T=M L \nabla U(L) \cdot \hat{\mathbf{u}}_{\perp} & +\sum_{i=1}^{n}\left[\nabla U\left(s_{i}\right) \cdot \hat{\mathbf{u}}_{\perp}-2(1+\dot{\theta}) \dot{s}_{1}\right] s_{i}+ \\
& +\sum_{i=n+1}^{2 n} D\left[\nabla U\left(s_{i}\right) \cdot \hat{\mathbf{u}}_{\perp}+2(1+\dot{\theta}) \dot{s}_{1}\right] s_{i}
\end{aligned}
$$

Note that if $\dot{\theta}>-1$, the Coriolis torque (proportional to $(1+\dot{\theta})$ on Eq. (19)) is always negative (i.e., inducing a clockwise rotation, opposite to the asteroid rotation). The limiting case $\dot{\theta}=-1$ corresponds to the scenario where the angular velocity of the siphon has the same magnitude as the angular velocity of the asteroid however with opposite direction: in this case, the global Coriolis torque vanishes. 


\subsection{Siphon dynamics}

The overall siphon dynamics are then described by the combination of the translational chain motion $s_{1}$ and the rotation $\theta$ :

$$
\begin{aligned}
n(1+D) \ddot{s}_{1} & =f_{r} \\
{\left[\sum_{i=1}^{2 n} m_{i} s_{i}^{2}+M L^{2}\right] \ddot{\theta} } & =T
\end{aligned}
$$

where $f_{r}$ and $T$ are the radial force and torque on the siphon, given by Eqs. (16) and 19 respectively. Note that the two equations of motion are coupled, being $f_{r}$ function of $\theta$ and $\dot{\theta}$, and $T$ a function of $s_{1}$ and $\dot{s}_{1}$. Again, it is emphasized that each $s_{i}$ can be written as a function of $s_{1}$ according to Eq. 12 , due to the hypothesis of rigid tethers connecting the buckets. From now on, when the context is clear, the subscript 1 will be omitted from the generalized coordinate $\dot{s}_{1}$.

Equations 200 are non-autonomous, non-linear and non-homogeneous differential equations and do not admit a closed-form solution. They can be numerically integrated from an initial state $\left\{s\left(t_{0}\right), \dot{s}\left(t_{0}\right), \theta\left(t_{0}\right), \dot{\theta}\left(t_{0}\right)\right\}$ and an initial CS mass $M\left(t_{0}\right)$. Let $\left\{s\left(t_{1}\right), \dot{s}\left(t_{1}\right), \theta\left(t_{1}\right), \dot{\theta}\left(t_{1}\right)\right\}$ be the state when $s=l$, i.e., when the top payload has reached the CS and a new empty bucket from the DS has reached the anchor point. It is assumed that the payload to be added to the chain has zero velocity within the rotating frame. By modelling this event as an inelastic collision, the new velocity of the chain $\dot{s}$ after a new payload is added is $\dot{s}\left(t_{1}\right)(n-1) / n$ (see Appendix A). Then, the new state after this event will be

$$
\left\{0, \frac{n-1}{n} \dot{s}\left(t_{1}\right), \theta\left(t_{1}\right), \dot{\theta}\left(t_{1}\right)\right\}
$$

A new integration can then be performed with the updated velocity and CS mass $\left(M\left(t_{1}\right)=M\left(t_{0}\right)+\right.$

$m_{p}$ ), and the process is iteratively repeated. Note that the translational degree of freedom $s$ is bounded between 0 and $l$. As a compact notation, the subscript 0 will be used in the following sections to indicate a variable at the beginning of the simulation.

\subsubsection{Siphon rotational damping}

The siphon rotational dynamics, Eq. 20b, can be rearranged as:

$$
I \ddot{\theta}+c \dot{\theta}+k(\theta)=-c
$$


where

$$
\begin{aligned}
I & =\sum_{i=1}^{2 n} m_{i} s_{i}^{2}+M L^{2} \\
c & =2 \dot{s}\left[\sum_{i=1}^{n} s_{i}-\sum_{i=n+1}^{2 n} D s_{i}\right] \\
k & =T_{c s}+\sum_{i=1}^{n}\left(\nabla U\left(s_{i}\right) \cdot \hat{\mathbf{u}}_{\perp}-2 \dot{s}_{1}\right) s_{i}+ \\
& +\sum_{i=n+1}^{2 n} D\left(\nabla U\left(s_{i}\right) \cdot \hat{\mathbf{u}}_{\perp}+2 \dot{s}_{1}\right) s_{i}
\end{aligned}
$$

The first and third term on the left-hand side of Eq. (22) represent the inertia and the stiffness of the siphon respectively, and they linearly increase with the CS mass $M$. The second term $c$, does not depend on the CS mass but on the current chain mass distribution and the chain velocity $\dot{\theta}$. Being $D<0$ by definition (Eq. (14)), the term within square brackets in Eq. (24) is always positive, for any choice of $n$ and $L$. Then, the sign of $c$ depends on the sign of the siphon radial velocity, which is positive if the orbital siphon effect is generated (i.e., $f_{r}>0$, Eq. (16)). Therefore, the term $c$ can be thought of as a damping term, responsible for attenuation of the siphon oscillation and it is due to the Coriolis forces associated with the rotation of the chain. This behaviour is typical of frictionless pendulums with moving mass (see, for example, Ref. [15]). Note that the term $c$ also appears at the right-hand-side of Eq. 222. Since $c>0$, the term $-c$ effectively represents a torque in the clockwise direction, which induces the chain to rotate opposite to the direction of the asteroid rotation. Therefore, the motion of the chain has a double effect on the system: (1) it attenuates the amplitude of the siphon oscillation and (2) forces the siphon to rotate clockwise.

As more mass is delivered to the CS and $M \gg 0$, the term $c$ (not depending on $M$ ) becomes negligible with respect to $I$ and $k$, therefore it is expected that the damping is gradually reduced over time. Although a formal stability analysis of the system is not performed in this paper, such qualitative comments will be helpful for interpretation of the numerical simulation results presented in Sect. 4 .

\subsection{Approximations for a continuous chain}

In the following sections a set of parameters are defined, where the LS and DS are approximated with continuous mass distributions with linear densities $\rho_{l s}=n m_{l s} / L$ and $\rho_{d s}=n m_{d s} / L$. In this 
scenario, the parameter $D$ can also be written as the ratio between the two densities $\rho_{d s} / \rho_{l s}$. It will be shown that such approximations can be exploited to quickly analyse the effects of the asteroid shape on the siphon dynamics.

\subsubsection{Siphon equilibrium length}

The siphon equilibrium length is defined as the length $L_{\text {min }}$ such that the overall radial force on the siphon $f_{r}$ is zero when the siphon orientation is fixed $(\dot{\theta}=0)$ for continuous mass distributions along the LS and DS. In such scenario the radial force given by Eq. 16) can be written as the integral

$$
\left.f_{r}\right|_{n \rightarrow \infty}=(1-D) \int_{0}^{L} \nabla U \cdot \hat{\mathbf{u}}_{r} d \sigma
$$

where the discrete variable $s_{i}$ is replaced with $\sigma$, which varies continuously in the range $[0, L]$ and represents the distance of a generic point on the (continuous) chain with respect to the anchor point. Then, $L_{\min }$ can be found by solving $\left.f_{r}\right|_{n \rightarrow \infty}=0$, i.e.,

$$
\int_{0}^{L_{\min }} \nabla U(\sigma) \cdot \hat{\mathbf{u}}_{r} d \sigma=0
$$

When $L=L_{\min }$ the gravitational and centrifugal-induced forces acting on the DS and LS balance, removing any orbital siphon effect. In general, Eq. 27) does not admit a close-form solution, due to the integrals in Eqs. (9), related to the gravitational component of the effective potential. However, if $\beta=\gamma=1$ (i.e., for a spherical asteroid), and $\theta=0$ then Eq. 27) simplifies to

$$
L_{\min }=\frac{1}{2}\left[\sqrt{9-8\left(1-\frac{1}{\omega^{2}}\right)}-3\right]
$$

which is the same result found in [7] for the minimum length of a siphon anchored to a spherical asteroid. Note that $L_{\min }$ is independent of the linear densities of the LS and DS, as the parameter $D$ does not appear. As in [7] it can be shown that if $L>L_{\min }$ then $f_{r}>0$ for any $n \geq 2$ (with $\left.s_{1}>l / 2\right)$. Therefore, to guarantee the siphon effect, it is necessary to ensure that $L>L_{\min }$ for the angle $\theta$ (or the range of angles) at which the siphon is operating.

Figure 4 shows $L_{\min }$ for $\phi_{A} \in[0,2 \pi]$ considering an asteroid period $2 \pi / \bar{\omega}=5 \mathrm{~h}$, assuming $\theta=0, \forall \phi_{A}$ and $\beta=\gamma=0.5$ (Fig. 4a), $\beta=\gamma=0.9$ (Fig. 4b). If the top of the siphon is contained within the shaded region then $\left.f_{r}\right|_{n \rightarrow \infty}<0$ and the siphon effect cannot be generated. Note that 


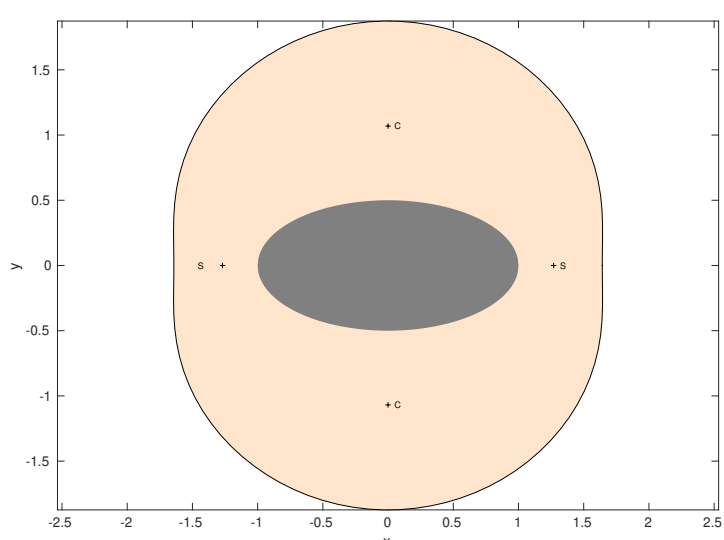

(a)

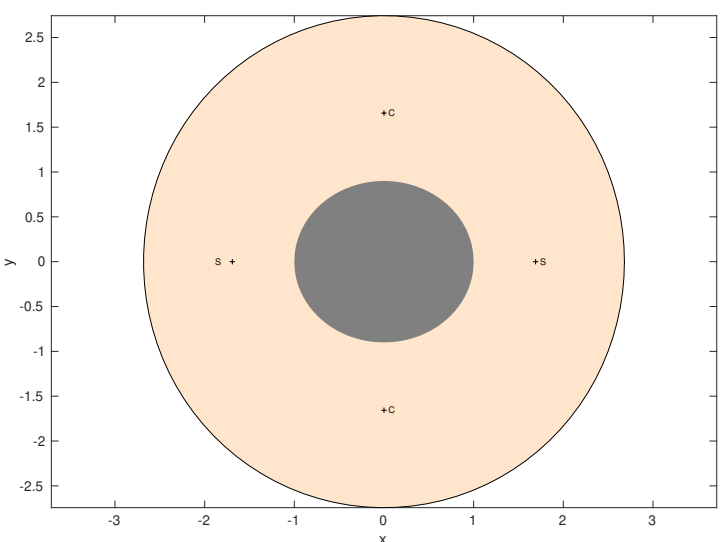

(b)

Figure 4: Representation of $L_{\min }$ assuming $\theta=0$ (black line at the boundary of the shaded region) for an asteroid with period $5 \mathrm{~h}$ and $\beta=\gamma=0.5$ (a), $\beta=\gamma=0.9$ (b). To guarantee the orbital siphon effect the chain length must extend beyond the shaded region. Center $(\mathrm{C})$ and Saddle (S) equilibrium points are also represented.

the shaded region contains the EPs and the minimum length in the region $\phi_{A} \approx \pi / 2$ is larger than that for $\phi_{A} \approx 0$ when the asteroid is more prolate.

Figures $5 \mathrm{a}$ and $5 \mathrm{~b}$ show the equilibrium length $L_{\min }$ as a function of the asteroid period for anchoring longitude $\phi_{A}=0$ (a), $\phi_{A}=\pi / 2$ (b) and a range of $\beta$ assuming $\gamma=\beta$ and the chain normal to the surface $(\theta=0)$ in both cases. As expected, the equilibrium length increases (almost linearly) with the asteroid rotational period. In fact, larger rotational periods will decrease the effect of the centrifugal-induced acceleration in the effective potential, thus increasing the altitude of the EPs and requiring longer chains for equilibrium. When the chain is anchored at $\phi_{A}=\pi / 2$ the equilibrium length becomes longer, as shown in Fig. 4 (the difference with respect to the case $\phi_{A}=0$ is more evident for more prolate ellipsoids). It can be shown that when $\gamma<\beta$ the equilibrium length decreases for a given period.

Figures $5 \mathrm{c}$ and $5 \mathrm{~d}$ show the equilibrium length as a function of the chain angle $\theta$ for anchoring longitude $\phi_{A}=0$ (left), $\phi_{A}=\pi / 2$ (right) and a range of $\beta$ assuming again $\gamma=\beta$. It is apparent that when $\theta \neq 0$ the equilibrium length increases (the plot is the same for $\theta \in[0,-\pi / 2]$ due to symmetries and the case with $\phi_{A}=\pi$ and $\phi_{A}=3 \pi / 2$ would be equivalent to $\phi_{A}=0$ and $\phi_{A}=\pi / 2$ respectively). Larger (smaller) asteroid densities will also increase (reduce) the magnitude of the gravitational acceleration thus increasing (reducing) the equilibrium length. 


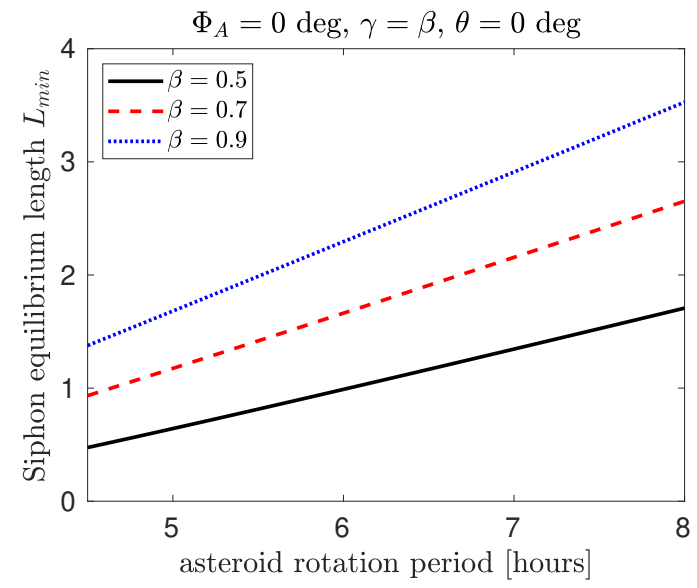

(a)

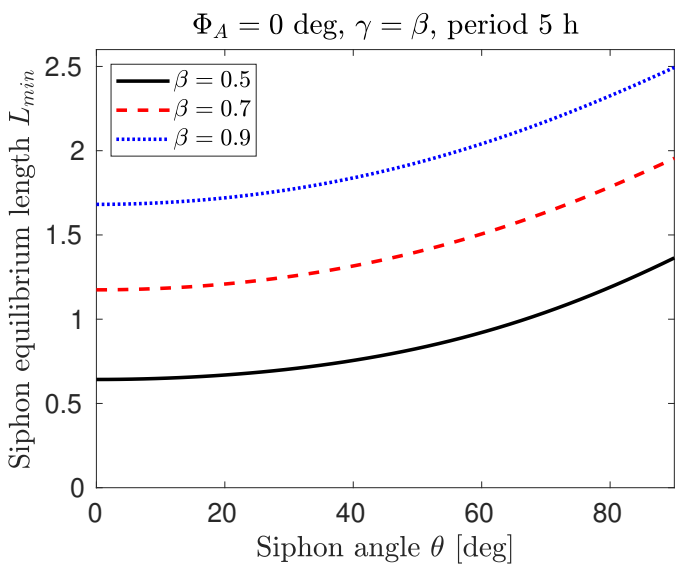

(c)

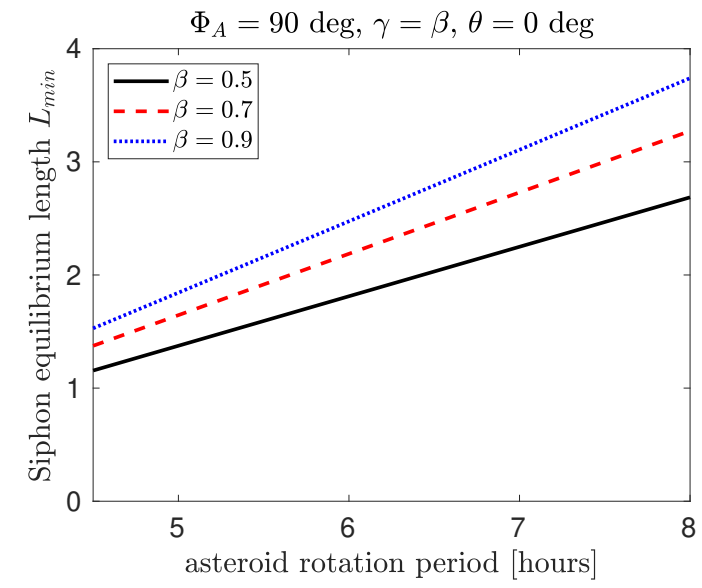

(b)

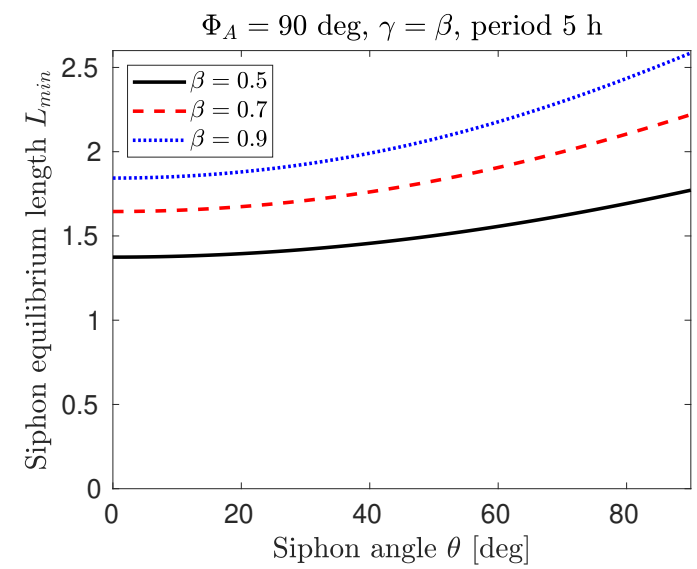

(d)

Figure 5: (a), (b): equilibrium length $L_{\min }$ as a function of the asteroid period for anchoring longitude $\phi_{A}=0$ (a) and $\phi_{A}=\pi / 2(\mathrm{~b})$ assuming $\theta=0$ in both cases. (c), (d): equilibrium length as a function of the chain angle $\theta$ for anchoring longitude $\phi_{A}=0$ (c) and $\phi_{A}=\pi / 2$ (d) assuming $\theta=0$ and a rotation period of $5 \mathrm{~h}$. 


\subsubsection{Siphon steady state velocity}

The siphon steady state velocity $v_{s s}$ is defined as the velocity of the chain when the time $t \rightarrow \infty$ for a given fixed $\theta$, using a continuous mass distribution for the chain. This parameter is known from earlier work for a discrete mass of distribution of $n$ masses [7]

$$
v_{s s}=\sqrt{\frac{2 W}{1-\left(\frac{n-1}{n}\right)^{2}}}
$$

where $W$ is the work per unit mass done by the gravitational and centrifugal-induced forces to pull the chain up by the distance between two consecutive payload masses $l$ :

$$
W=\int_{0}^{l} a d \sigma
$$

with $a$ being the acceleration of the chain. For a continuous mass distribution $n \rightarrow \infty, l \rightarrow L / n \rightarrow 0$ and the work per unit mass becomes

$$
W=a \frac{L}{n}
$$

where the acceleration can be written as

$$
a=\frac{\left.f_{r}\right|_{n \rightarrow \infty}}{(1+D) L}
$$

with $\left.f_{r}\right|_{n \rightarrow \infty}$ given by Eq. 26. Note that the denominator of Eq. 232 is the overall mass of the siphon under the continuous mass distribution approximation, comprising the LS and DS. Substituting Eq. 231 into Eq.29) and calculating the limit for $n \rightarrow \infty$, the steady state velocity can be written as:

$$
v_{s s}=\sqrt{\frac{1-D}{1+D}\left(\int_{0}^{L} \nabla U(s) \cdot \hat{\mathbf{u}}_{r} d s\right)}
$$

When $D=1$ (i.e., the LS is unloaded) it can be seen that $v_{s s}=0$. Similarly, if the chain length is equal to the equilibrium length then, by Eq. 27) $\int_{0}^{L} \nabla U \cdot \hat{\mathbf{u}}_{r}=0$ and again $v_{s s}=0$. Conversely, when the ratio $m_{p} / m_{b} \gg 1(D \rightarrow 0)$ the chain velocity is maximum, for a given asteroid, chain length, anchor longitude and angle $\theta$. The terms including $\dot{\theta}$ do not appear in Eq. (33) as the steady state velocity is defined for fixed $\theta$.

It is instructive to simplify Eq. 33 in case of a spherical asteroid $(\beta=\gamma=1)$, which admits 
the closed-form solution

$$
v_{s s}^{\text {sphere }}=\sqrt{\frac{1-D}{1+D}\left[\frac{1}{2}\left(r_{L}^{2}-1\right)-\frac{1}{\omega^{2}} \frac{r_{L}-1}{r_{L}}\right]}
$$

where $r_{L}=\sqrt{1+L^{2}+2 L \cos \theta}$ is the distance between the CS and the center of the asteroid. Setting $D=0$ and taking chain normal to the asteroid surface $(\theta=0)$ Eq. (34) reduces to the value found in 7 ]

$$
v_{s s}^{\text {sphere }}=\sqrt{\frac{L}{2}\left(2+L-\frac{2}{(1+L) \omega^{2}}\right)} .
$$

\subsubsection{Siphon equilibrium angle}

This parameter is defined as the angle $\theta_{\text {eq }}$ such that the overall torque $T$ with respect to the anchor point is zero, when the chain is approximated with a continuous mass distribution and $\dot{s}=v_{s s}$. As it will be shown in Sect. 4. $\theta_{\mathrm{eq}}$ is an indicator of the average oscillation $\theta$ of the siphon. From Eq. 19], substituting the sums with integrals, $\theta_{\text {eq }}$ can be found as the solution of

$$
\frac{L^{2}}{2}(D-1) v_{s s}\left(\theta_{\mathrm{eq}}\right)+L^{2} M^{*} \nabla U(L) \cdot \hat{\mathbf{u}}_{\perp}+(1+D) \int_{0}^{L} \nabla U(\sigma) \cdot \hat{\mathbf{u}}_{\perp} s d \sigma=0
$$

where $v_{s s}\left(\theta_{\text {eq }}\right)$ is the steady state velocity calculated at the equilibrium angle. Here $M^{*}$ is the mass of the CS scaled with respect to the total mass on the LS of the siphon.

\section{Results}

In this section the orbital siphon dynamics (Eqs. 20) are integrated for a given choice of asteroid and siphon parameters. Unless explicitly specified in the text, the baseline simulation parameters are listed in Table 1 .

Figure 6 shows the angle $\theta$ (Fig. 6a), siphon angular velocity $\dot{\theta}$ (Fig. 6b), chain velocity $\dot{s}$ (Fig. 6c) and CS mass $M$ (Fig. 6d as a function of time for two different initial states: $\left\{s_{0}, \dot{s}_{0}, \theta_{0}, \dot{\theta}_{0}\right\}=$ $\{0,0,0,0\}$ (case A) and $\left\{s_{0}, \dot{s}_{0}, \theta_{0}, \dot{\theta}_{0}\right\}=\left\{0, v_{s s}, \theta_{\text {eq }}, 0\right\}$ (case B). In case A, the siphon is initially aligned with the local vertical and is at rest. In case $B$, the siphon is initialized at its equilibrium angle $\theta_{\text {eq }}$ for the initial CS mass $M_{0}$, and its initial velocity $\dot{s}_{0}$ is the steady state velocity at $\theta_{\text {eq }}$. Figures 6 a and $6 \mathrm{c}$ also show the equilibrium angle $\theta_{\text {eq }}$ (blue, dashed line) for the current CS mass at time $t$ and the steady state velocity $v_{s s}$ respectively. Some relevant information can be inferred: 


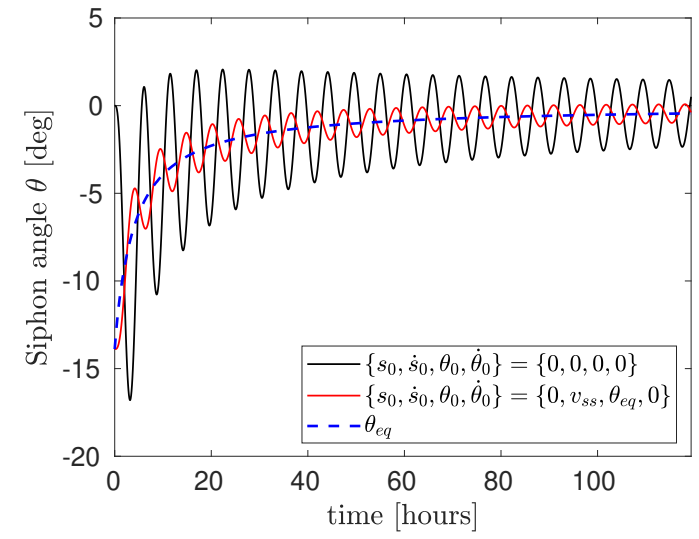

(a)

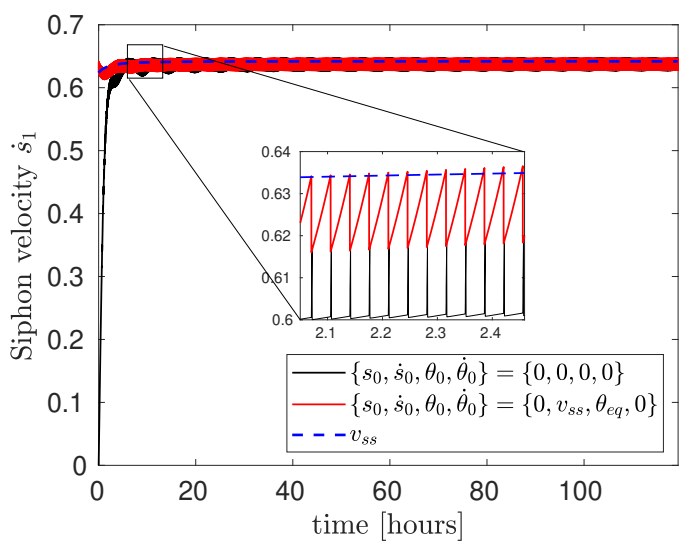

(c)

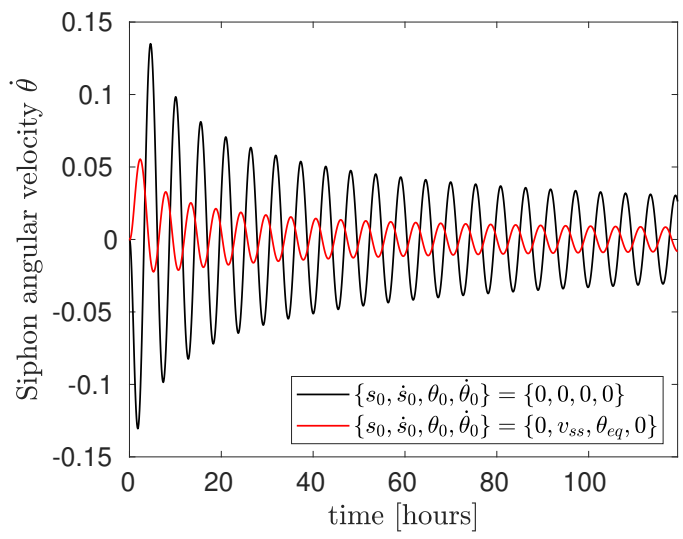

(b)

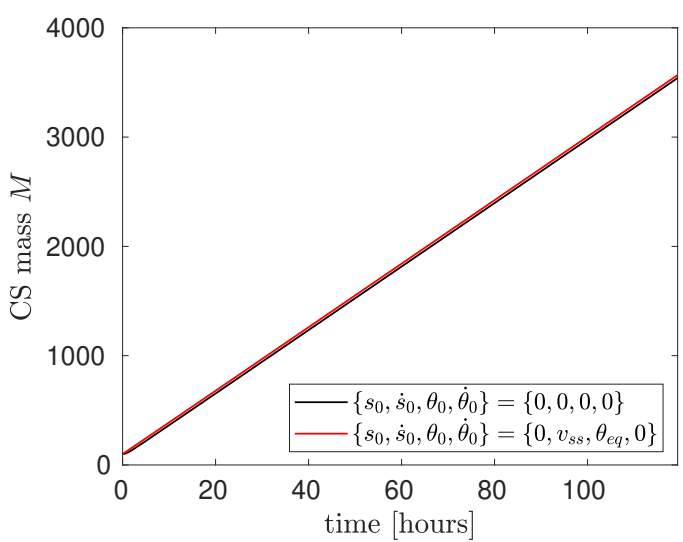

(d)

Figure 6: Siphon angle $\theta$ (a), non-dimensional siphon angular velocity $\dot{\theta}$ (b), non-dimensional chain velocity $\dot{s}$ (c) and non-dimensional CS mass $M,(\mathrm{~d})$, as a function of time. The blue dotted curve in Figs. 6a and 6c represent the instantaneous equilibrium angle (Eq. 36 and the steady state velocity (Eq. 33 ) respectively.

Table 1: Baseline simulation parameters. All units are non dimensional. The selected siphon length corresponds to $1.5 L_{\min }$. The parameter $\omega$ corresponds to an asteroid with density $\rho=2.5 \mathrm{~g} \mathrm{~cm}^{-3}$ rotating with period $2 \pi / \bar{\omega}=5 \mathrm{~h}$.

\begin{tabular}{llcc}
\hline \multirow{3}{*}{ Asteroid } & Angular velocity & $\omega$ & 0.84 \\
& Extent & $\beta$ & 0.5 \\
& Length & $\gamma$ & 0.5 \\
\hline \multirow{4}{*}{ Siphon } & Initial CS mass & $M_{0}$ & 0.96 \\
& Number of payloads & $2 n$ & 100 \\
& Initial state & $\left\{s_{0}, \dot{s}_{0}, \theta_{0}, \dot{\theta}_{0}\right\}$ & $\{0,0,0,0\}$ \\
& DS to LS mass ratio & $D$ & 0 \\
& Anchor longitude & $\phi_{A}$ & 0 \\
\hline
\end{tabular}


1. The siphon exhibits a damped oscillatory behaviour. In both cases, the siphon angle $\theta$ oscillates about the equilibrium angle $\theta_{\mathrm{eq}}$, and $\theta_{\mathrm{eq}} \rightarrow 0$, as $t \rightarrow \infty$. The amplitude of the oscillations is smaller if the siphon is initialised at the equilibrium angle (case B).

2. The period of the oscillations is comparable with the asteroid period.

3. The chain velocity changes at two different frequencies (see Fig. 6c): a lower frequency is associated with the variation of the siphon angle $\theta$ (which regulates the magnitude of the net radial force on the chain) while the higher frequency is due to the bucket refilling. Note that at each bucket refilling, the chain velocity changes according to Eq. (21). In the long term, the chain velocity does not diverge but oscillates about an average value which is well approximated by $v_{\mathrm{ss}}$.

4. The CS mass varies almost linearly over time and it is slightly larger in case B due to the larger velocity of the chain at the beginning of the simulation.

As anticipated in Sect 3.3.1 the damping effect is progressively reduced as the mass of the CS increases and the siphon exhibits stable oscillations about $\theta_{\text {eq }}$. Figure 7 shows the results of a longer simulation (33 days) in the phase space $(\theta, \dot{\theta})$ for case A $7 \mathrm{a}$ and $\mathrm{B} 7 \mathrm{~b}$. The initial state at $t=0$ is marked with a cross. As expected, the equilibrium of the system gradually shifts towards $(\theta, \dot{\theta})=(0,0)$ and the damping effect is progressively reduced over time.

Figure 8 shows the siphon angle and chain velocity as a function of time for variable initial CS masses $M_{0}$. The initial CS mass influences the amplitude of the oscillations, in particular, the initial overshoot. As the initial CS mass increases, the Coriolis torque becomes negligible with respect to the centrifugal-induced torque caused by the CS, thus reducing the magnitude of $\theta$.

Figure 9 shows the siphon angle and chain velocity as a function of time for variable mass ratios $D$. The mass ratio $D$ mainly affects the chain velocity. As $D$ increases, the ratio between the mass of the payloads and the mass of the buckets decreases as dictated by Eq. (14), thus reducing the radial force on the chain (Eq. (16)), eventually reducing its velocity. As a consequence, also the steady state velocity decreases, as predicted by Eq. 33.

Figure 10 shows the siphon angle and chain velocity as a function of time for $n=20, n=50$ and $n=80$. The number of payloads affects the magnitude of the Coriolis torque on the chain: more payloads on the chain will lead to larger oscillations. The chain velocity is almost unaffected 


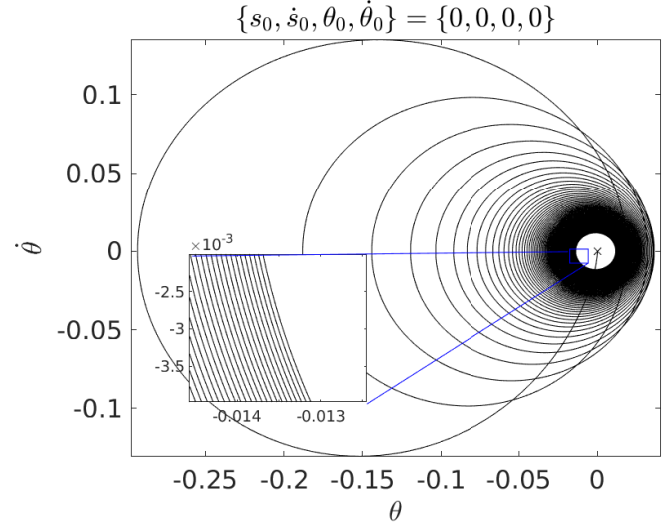

(a)

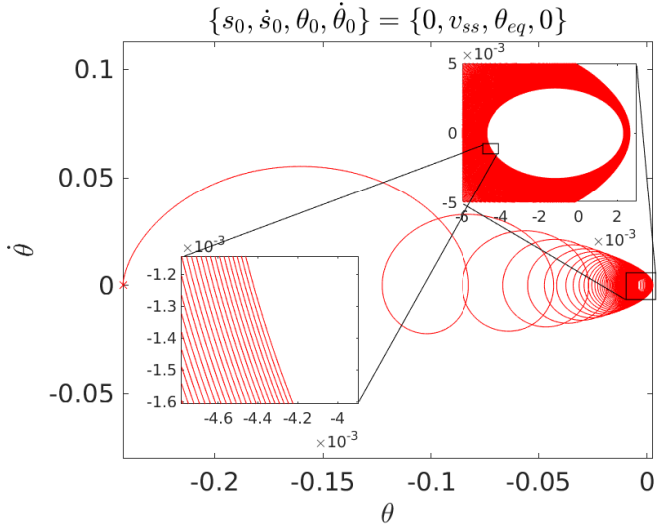

(b)

Figure 7: Siphon trajectories in the phase space $(\theta, \dot{\theta})$ for a 33 days simulation, for different initial conditions, $\left\{s_{0}, \dot{s}_{0}, \theta_{0}, \dot{\theta}_{0}\right\}=\{0,0,0,0\}$ (a) and $\left\{s_{0}, \dot{s}_{0}, \theta_{0}, \dot{\theta}_{0}\right\}=\left\{0, v_{s s}, \theta_{\text {eq }}, 0\right\}$ (b). The insets are close-up views near $(\theta, \dot{\theta}) \approx$ $(0,0)$.

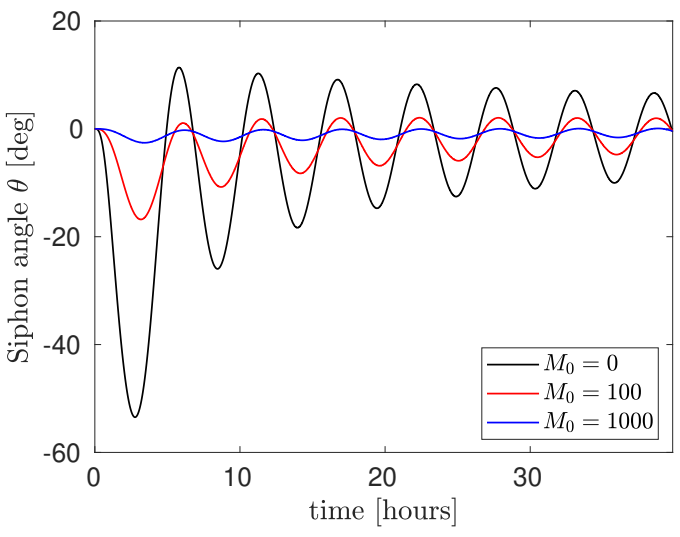

(a)

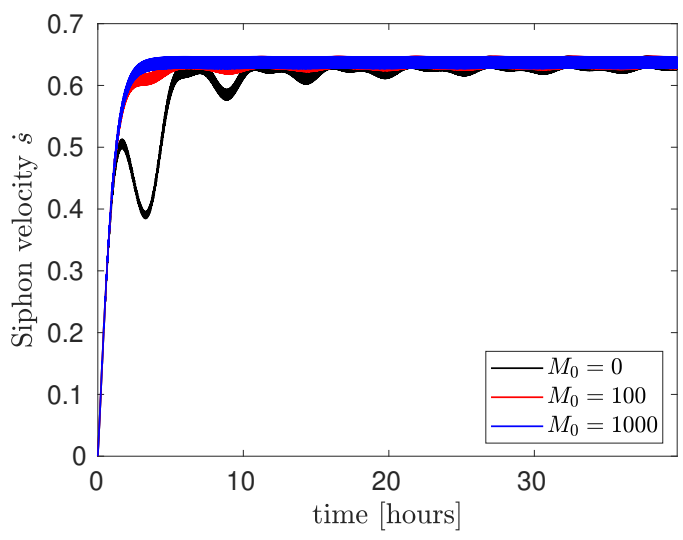

(b)

Figure 8: Siphon angle $\theta$ (a) and chain velocity $\dot{s}(\mathrm{~b})$ as a function of time for different CS mass at $t=0, M_{0}=0$, $M_{0}=100, M_{0}=1000$. 


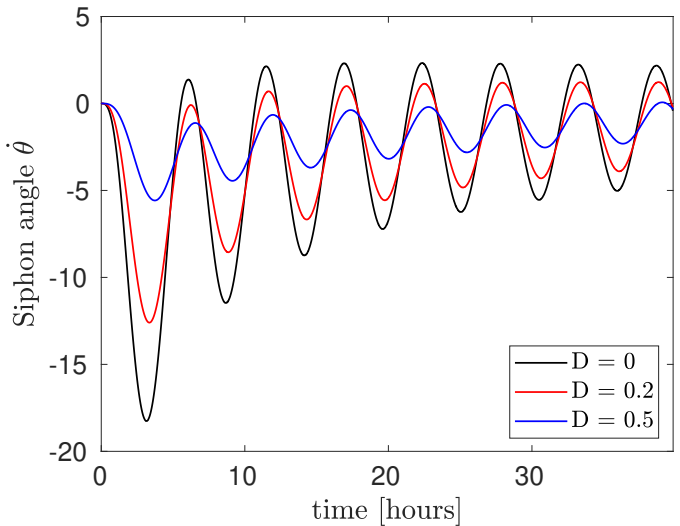

(a)

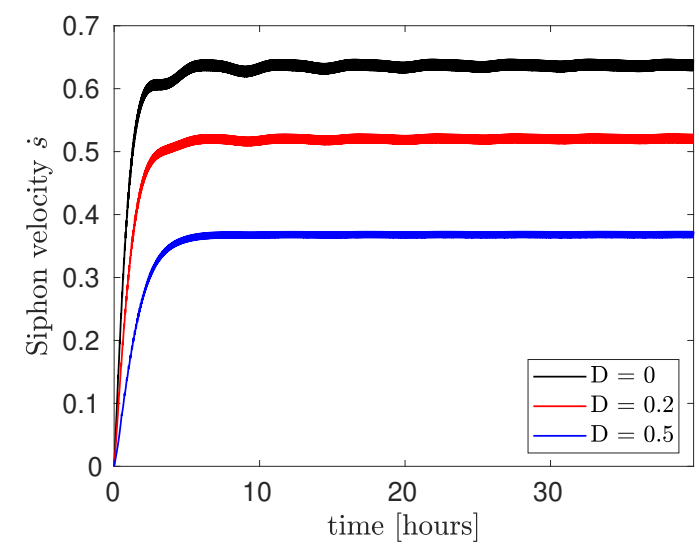

(b)

Figure 9: Siphon angle $\theta$ (a) and chain velocity $\dot{s}$ (b) as a function of time for $D=0, D=0.2$ and $D=0.5$.

even though as $n$ increases the variation of $\dot{s}$ between consecutive bucket-refilling events slightly decreases (Fig. 10b): in fact, by increasing $n$ with a fixed chain length, the distance $l$ between consecutive buckets decreases and therefore the frequency of payload refilling increases.

Figure 11 shows the siphon angle and chain velocity as a function of time for the two siphon lengths $L=1.2 \mathrm{~L}_{\min }$ and $L=1.7 \mathrm{~L}_{\min }$. A larger chain length will cause a larger radial velocity. Note that, although the oscillations have slight different phases, the period of the oscillations does not vary significantly between the two cases.

It has been noted that the parameters $\theta_{\mathrm{eq}}$ and $v_{\mathrm{ss}}$ are good approximations of the siphon angle and the chain velocity at steady state. Moreover, the CS mass varies almost linearly with time. Therefore, it is instructive to investigate how the equilibrium angle and chain velocity vary as a function of the CS mass $M$, by varying other parameters such as the asteroid shape and the anchor longitude. Figure 12 illustrates the variation of $\theta_{\mathrm{eq}}$ and $v_{\mathrm{ss}}$ as a function of the CS mass for a range of chain lengths $L$ and anchor longitudes $\left(\phi_{A}=0, \phi_{A}=\pi / 4, \phi_{A}=\pi / 2\right)$, taking an asteroid with $\beta=\gamma=0.3$ and other parameters as indicated in Table 1 . Figure 13 , shows the same parameters, with $\beta=\gamma=0.8$. The siphon length is here chosen as a function of the minimum siphon length $L_{\min }$, calculated for $\theta=0\left(1.1 L_{\min }\right.$ (black curve), $1.5 L_{\min }$ (dashed red curve) and $2.5 L_{\min }$ (dotted blue curve)). To guarantee impact avoidance with the surface of the asteroid, the equilibrium angle $\theta_{\text {eq }}$ cannot exceed the threshold angle $\theta_{\text {lim }}$, which depends on the anchor location and $\beta$. The parameter $\theta_{\lim }$ is defined as the angle between $O A$ and the tangent to the anchor 


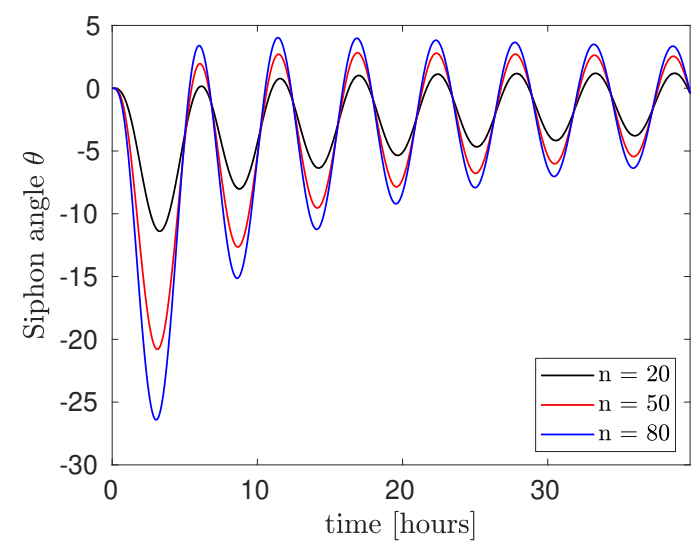

(a)

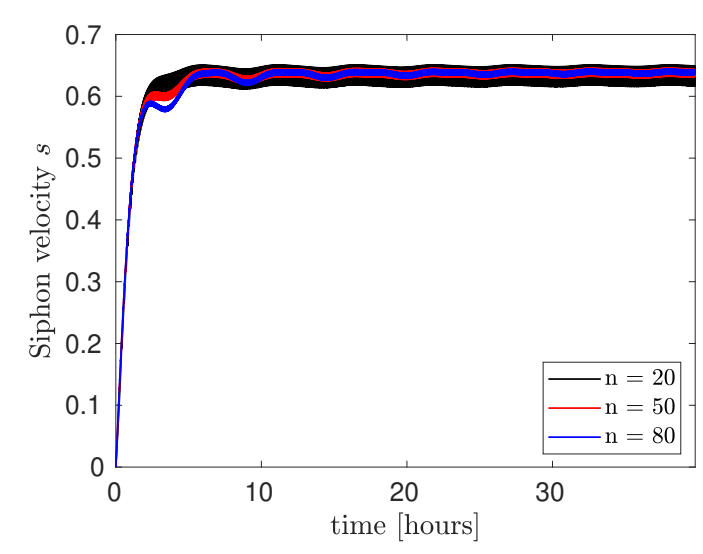

(b)

Figure 10: Siphon angle $\theta$ (a) and chain velocity $\dot{s}$ (b) as a function of time for $n=20, n=50$ and $n=80$.

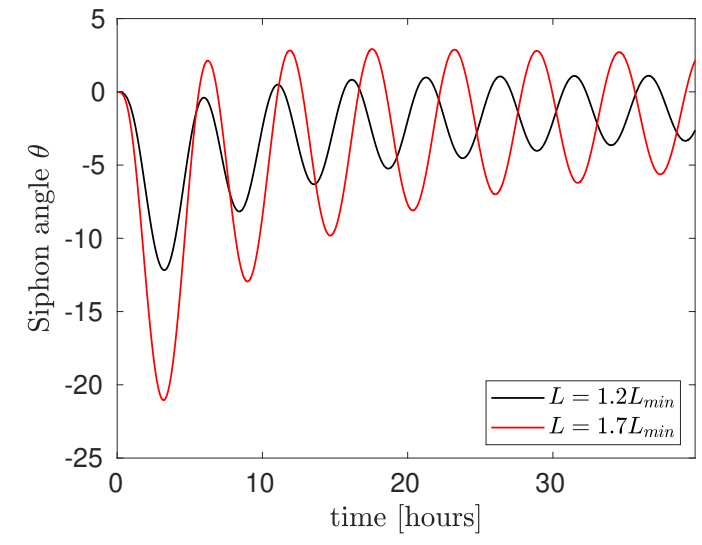

(a)

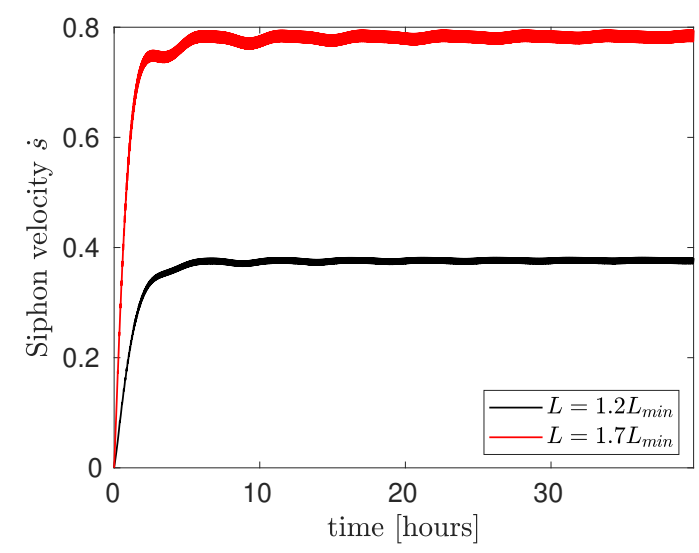

(b)

Figure 11: Siphon angle $\theta$ (a) and chain velocity $\dot{s}(\mathrm{~b})$ as a function of time for $L=1.2 L_{\min }$ and $L=1.7 L_{\min }$. 


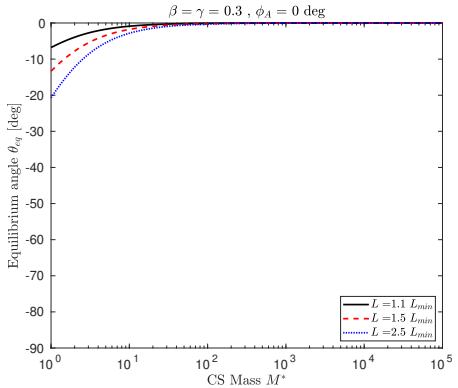

(a)

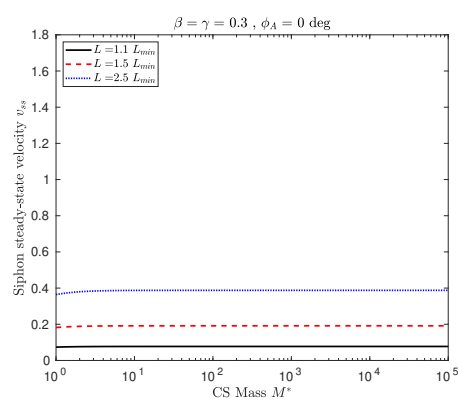

(d)

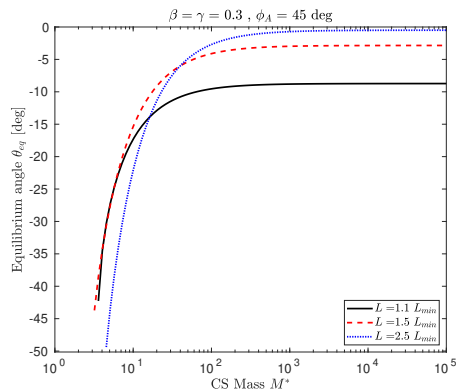

(b)

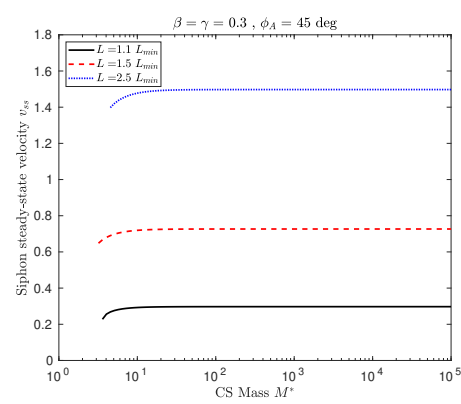

(e)

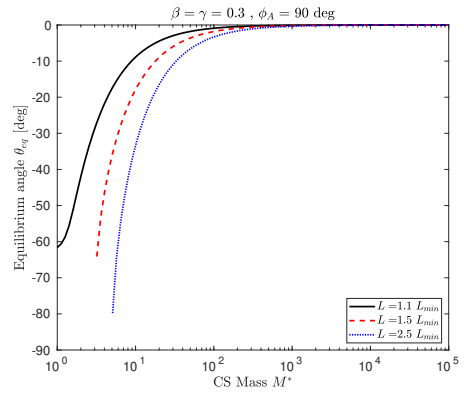

(c)

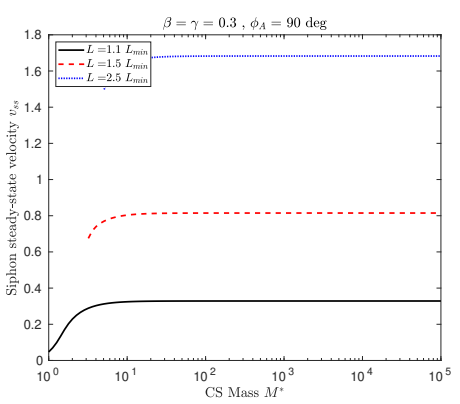

(f)

Figure 12: Equilibrium angle $\theta_{\mathrm{eq}}$ as a function of the CS mass $M^{*}$, for a range of anchor longitude and $\beta=\gamma=0.3$. 


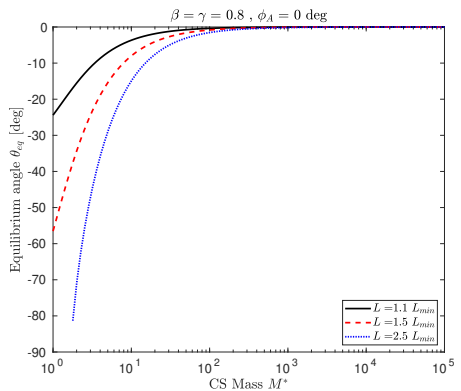

(a)

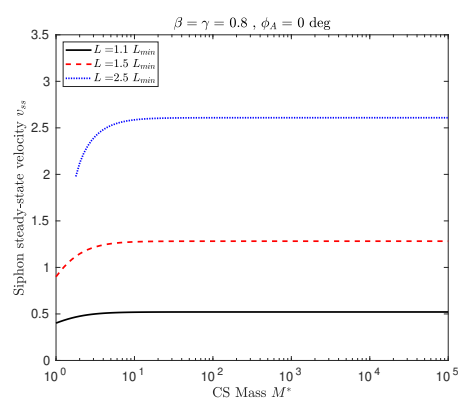

(d)

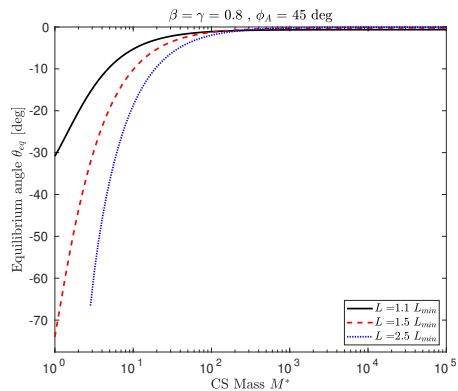

(b)

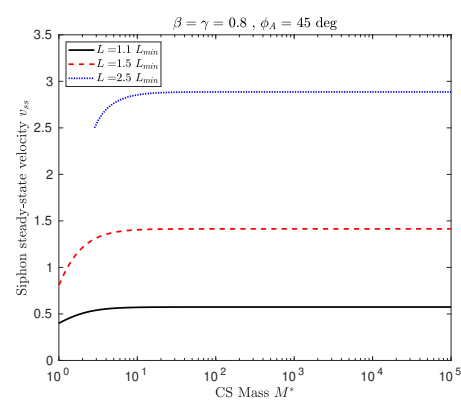

(e)

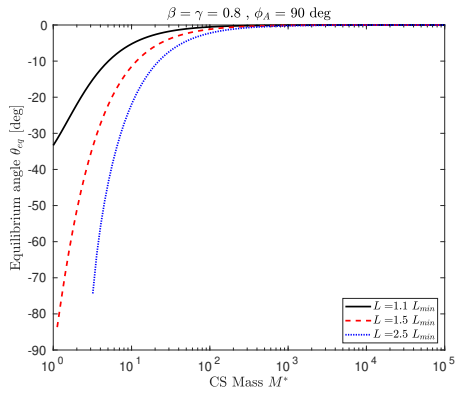

(c)

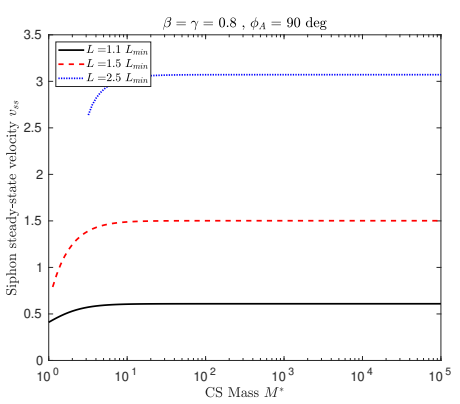

(f)

Figure 13: Equilibrium angle $\theta_{\mathrm{eq}}$ as a function of the CS mass $M^{*}$, for a range of anchor longitude and $\beta=\gamma=0.8$. 


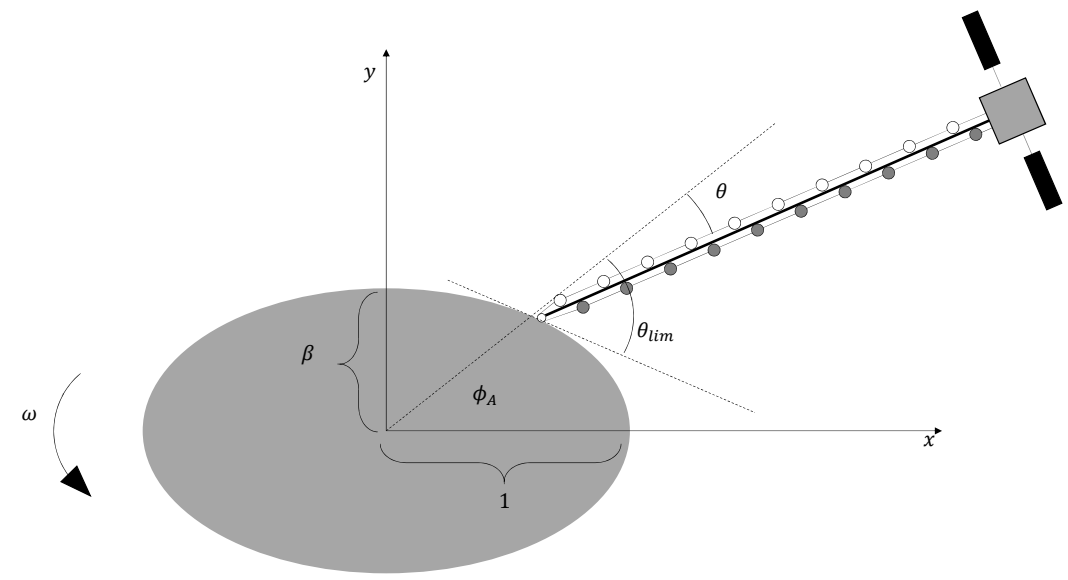

Figure 14: Maximum siphon angle $\theta_{\text {lim }}$.

point in the equatorial plane, in the direction shown in Fig. 14 (the complementary to $\theta_{\text {lim }}$ is not considered, since the siphon will rotate clockwise due to the Coriolis torque). For a siphon anchored at the longest or shortest equatorial end, $\theta_{\lim }=-\pi / 2$ independent of $\beta$, whereas for $\phi_{A}=\pi / 4$, $\theta_{\lim } \approx-50 \mathrm{deg}$ when $\beta=0.3$ and $\theta_{\lim } \approx-70 \mathrm{deg}$ when $\beta=0.8$. In all the cases represented, the equilibrium angle decreases as the CS mass increases and $v_{\mathrm{ss}}$ is constant when the ratio $M^{*}$ between the CS mass and the LS of the siphon is larger than $10^{2}$. In particular, for $\phi_{A}=0$ or $\phi_{A}=\pi / 2, \theta_{\text {eq }} \rightarrow 0$ as the CS mass increases, and this holds for any $L>L_{\min }$ and does not depend on the asteroid shape. Note that at these anchor locations, the direction $\theta=0$ corresponds to the local vertical. Conversely, the effect of the asteroid shape influences $\theta_{\text {eq }}$ for intermediate anchor locations: the equilibrium angle for large values of the CS mass decreases for prolate bodies and this effect is enhanced if the siphon length is close to the equilibrium length (see Figs. 12b and 13b The equilibrium angle is not defined for some small values of $M^{*}$. In these cases, the siphon might exceed $\theta_{\text {lim }}$ during the initial overshoot and impact onto the asteroid surface. For longer chains, a larger initial CS mass is required to ensure $\left|\theta_{\text {eq }}\right| \leq\left|\theta_{\lim }\right|$. Therefore, for a given initial CS mass the siphon cannot be arbitrarily long: it is possible to define an upper bound on the siphon length that guarantees impact avoidance with the surface. Note that a lower bound also exist, to ensure that $L>L_{\min }$.

In summary, the following remarks can be made:

- An orbital siphon effect is generated due to the larger mass on the LS: the buckets on the 
LS are therefore pulled towards the CS while buckets on the DS cycle back to the anchor for refilling.

- The minimum siphon length required to generate the siphon effect depends on the anchor location. Smaller chains can be used when the siphon is anchored at the longest equatorial end.

- The orbital siphon effect introduces torques with respect to the anchor point, inducing damped oscillation of the chain.

- The radial velocity of the chain does not diverge, although a positive force is always acting on the LS. This is due to conservation of linear momentum during the bucket refilling process.

- The behaviour of the discrete chain can be approximated by a continuous mass distribution. This allows an estimation of the equilibrium angle and the chain radial velocity as a function of the CS mass and asteroid shape. The equilibrium angle approaches a constant value when the mass of the CS is large. In particular, if the siphon is anchored at the longest or smallest equatorial end, the siphon tends to align with the local vertical.

- By increasing the siphon length, the radial velocity of the chain increases and therefore the rate of payload material released to the CS also increases. As expected, larger chain velocities are achieved when the ratio $D \rightarrow 0$, i.e., when the mass of the buckets becomes negligible with respect to the mass of the payloads. In dimensional units, for siphon lengths comparable with the asteroid largest semi-major axis, the chain velocity is on the order of centimetres per second. For example, taking the asteroid Bennu (details in Sect. 5, Table 2), a siphon with length $L=0.6 \bar{\alpha}$ has a radial velocity of approximately $2.5 \mathrm{~cm} \mathrm{~s}^{-1}$.

\section{Transfers to equilibrium points}

It has been shown that as the siphon delivers material to the CS it will eventually reach a condition of stable oscillation and the velocity of the chain does not diverge. Due to the requirement $L>L_{\min }$ a released mass cannot intersect either the asteroid or any EP, and will eventually escape from the asteroid. This is a useful scenario if the material has to be sent to a higher energy orbit or to escape. 
Table 2: Bennu physical parameters [17.

\begin{tabular}{ccc}
\hline & $\bar{\alpha}$ & $282.5 \mathrm{~m}$ \\
Extent & $\bar{\beta}$ & $267.5 \mathrm{~m}$ \\
& $\bar{\gamma}$ & $254 \mathrm{~m}$ \\
Period & $2 \pi / \bar{\omega}$ & $4.297 \mathrm{~h}$ \\
Density & $\rho_{A}$ & $1.26 \mathrm{~g} \mathrm{~cm}^{-3}$ \\
\hline
\end{tabular}

The existence of equilibrium points, however, offers scope for further analysis. It has been shown in Sect. 2.1 that if the period of the asteroid is above a critical value depending on its shape, the CEPs are stable. Under such conditions, these points could be used in principle as gravitational depots for material. Taking advantage of these equilibria may therefore be useful in a large-scale mining scenario. For example, CEPs might host an orbiting catcher, receiving material from the siphon, for temporary storage or further processing.

Transfer of material to EPs may also be beneficial if the mined asteroid is a rubble pile with low cohesive strength [16, thus reducing the anchor force required to hold the orbital siphon to the asteroid surface. A conservative estimate of the required anchor force, assuming the CS mass $\bar{M}$ is much larger than the mass of the siphon, is $\bar{M} \bar{\omega}^{2}(\bar{\alpha}+\bar{L})$, i.e., the centrifugal-induced force acting on the CS, for anchoring at the longest equatorial end $\left(\phi_{A}=0\right)$ and $\theta=0$. For example, for a siphon with length $L=L_{\min }$ anchored at the asteroid Bennu (Table 2), the anchor force (in Newtons) scales as $10^{-6} \bar{M}$. Achievable anchor forces on rubble piles are on the order of $10 \mathrm{~N}$ for cohesion levels of $10 \mathrm{~Pa}$ [5]. In a large scale mining scenario, where the processed mass is larger than $10^{3}$ tonnes, the required force to keep this mass at the CS would be on the order of $10^{2} \mathrm{~N}$, hence one order of magnitude larger then the value suggested in Ref. [5]. The reader is referred to Ref. 12] for additional details on possible anchor devices for the orbital siphon.

A possible mechanism to deliver material to the CEPs is by employing a mass driver from the surface. Although the energy requirements are expected to be very small (due to the low-gravity on a typical near-Earth asteroid) it will be shown that the orbital siphon mechanism can offer in principle a more energy efficient way to deliver payloads to the CEPs.

As an example, the analysis proposed in the following sections is referred to the asteroid Bennu which has stable CEPs under the triaxial ellipsoid model. 


\subsection{Material transfer from the surface to a stable CEP}

The minimum amount of energy required to send material to any location around the asteroid from a given position at the surface can be derived analytically. In particular, let $E_{0}$ be the total energy per unit mass at the initial location of the material and let $E_{c}$ be the energy per unit mass at the CEPs (note that the two CEPs have the same energy due to symmetry in the ellipsoid model). If a change in velocity $\Delta v$ is applied at the initial point then, in order to intersect the CEP, the energy of the transfer trajectory should satisfy

$$
E_{0}+\frac{1}{2} \Delta v^{2} \geq E_{c}
$$

If Eq. (37) is not satisfied, then the position of the CEPs would be inside the forbidden region associated with the zero velocity curve of the transfer trajectory. Using dimensional units applied to the asteroid Bennu, a transfer from the longest end of the ellipsoid to the CEP requires $\Delta \bar{v} \geq$ $6.1 \mathrm{~cm} \mathrm{~s}^{-1}$, whereas a transfer from the shortest end to the CEP requires $\Delta \bar{v}>5.9 \mathrm{~cm} \mathrm{~s}^{-1}$. Let $\Delta \bar{v}_{\text {direct }}^{\min }=5.9 \mathrm{~cm} \mathrm{~s}^{-1}$ be the minimum of these two values, which will be used in later analysis.

Figure 15 shows an example of such trajectory departing from different longitudes. The trajectories are obtained by solving a boundary value problem, controlling the initial $\Delta v$ at the departure on the surface. Here, $\Delta \bar{v}_{1}$ represents the change in velocity required to insert the particle into the trajectory, whereas $\Delta \bar{v}_{2}$ is the change in velocity required to stop the particle at the equilibrium point.

\subsection{Exploiting siphon dynamics to transfer material to a stable CEP}

The proposed strategy is to exploit the siphon dynamics to induce oscillations to the chain in order to increase the energy of the CS and make the CEP accessible.

A possible method to avoid the intrinsic damping observed in Sect. 4 is to stop the chain during the counter-clockwise rotation of the siphon, to avoid the Coriolis torque which would reduce the oscillation amplitude. In particular, for a siphon anchored at $\phi_{A}=0$ with $\theta_{0}=0$ :

1. While the LS delivers material to the CS, the Coriolis torque will induce a clockwise rotation $(\dot{\theta}<0)$.

2. When $\dot{\theta}=0$ the chain is stopped, so $\dot{s}=0$. 


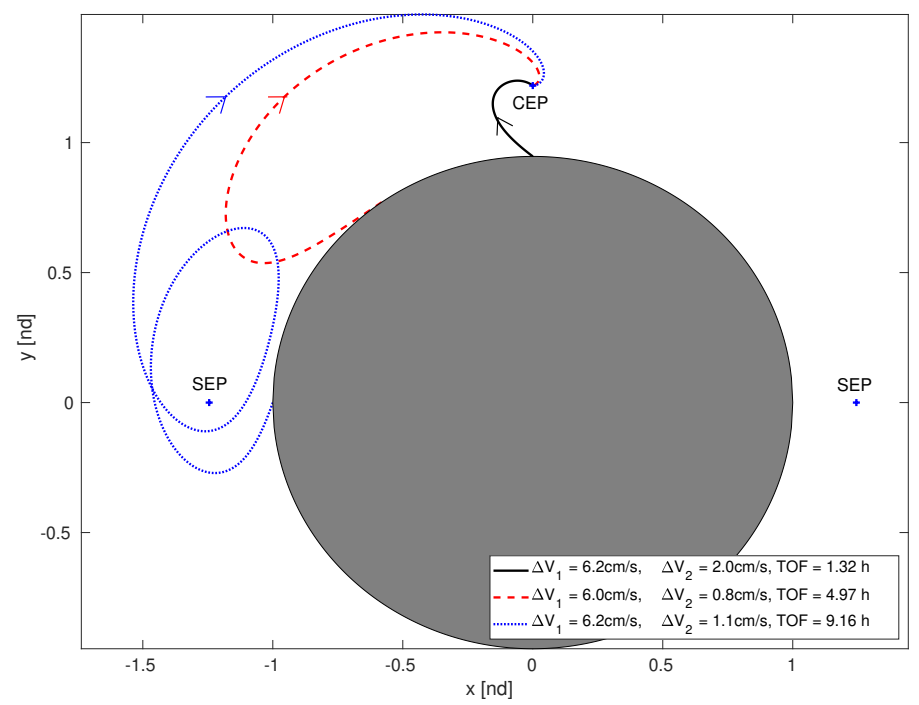

Figure 15: Example of low- $\Delta v$ transfer trajectories with a range of time of flight (TOF) from the surface of the asteroid Bennu to one of the two CEPs.

3. As no Coriolis torque is now acting on the siphon (due to the braked chain), the siphon operates like a standard undamped pendulum, reaching $\dot{\theta}=0$, without reducing the amplitude.

4. When $\dot{\theta}=0$ the chain brake is now released an the process repeated.

The maximum velocity which can be reached by the CS depends on the maximum amplitude of the chain $\theta_{\max }$ and on the length of the chain $L$. The maximum amplitude $\theta_{\max }$ must guarantee $L>L_{\min }\left(\theta_{\max }\right)$ where $L_{\min }\left(\theta_{\max }\right)$ is the equilibrium length calculated at $\theta=\theta_{\max }$. In any case, $\theta_{\max }<\pi / 2$ is necessary to avoid contact between the siphon and the asteroid surface. The length $L$ is chosen such that, when $\theta=0$, the CS velocity vector is tangent to a periodic orbit (PO) passing in close proximity to the CEPs. The PO is selected from the family displayed in Fig. 16a, These orbits are direct POs generated using standard differential correction algorithms. Suitable initial conditions were found via Ref. [14 and a continuation method was used to extend the family. Figure 16a shows some of the POs passing in close proximity to the CEPs. The orbits of this family are particularly interesting as they cross the $x$-axis with $\dot{y}=0$, and therefore they match the velocity direction of the CS when $\theta=0$. In particular, the trajectory represented in red, intersects both CEPs. This PO, with a period of $12.62 \mathrm{~h}$, is selected as a candidate transfer trajectory which will deliver material from the CS to one of the two CEP, where an orbiting catcher is envisaged to capture the transferred material. It can be shown, using the stability index method 


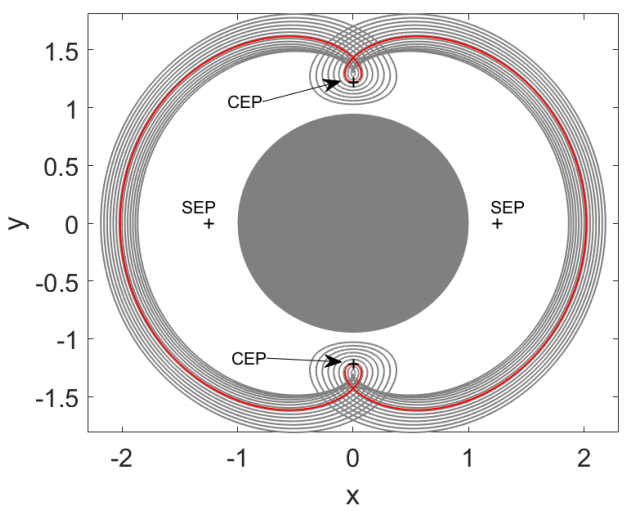

(a)

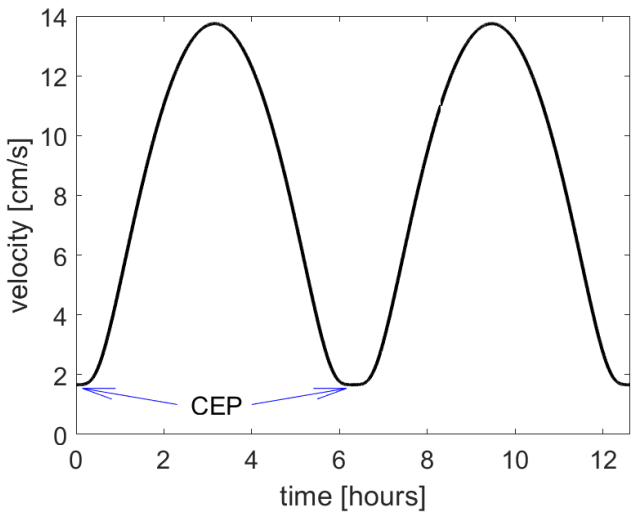

(b)

Figure 16: (a): Family of periodic orbits around Bennu. The trajectory intersecting the two CEPs is marked in red. (b): velocity of the periodic orbit intersecting the CEPs. Lower value of the velocity are reached at the intersection with the CEPs.

explained in Ref. [18, that this periodic orbit is stable. The velocity of this PO as a function of time is represented in Fig. $16 \mathrm{~b}$ using dimensional units. The maximum velocity $\bar{v}_{p o}=13.8 \mathrm{~cm} \mathrm{~s}^{-1}$ is reached at the crossing with the $x$-axis, whereas the lowest velocity is reached at the intersection with the two CEPs. The selected PO crosses the $x$-axis at $x=2.02$ and therefore, with the siphon anchored at $\phi_{A}=0$, the siphon length must be $L=2.02-\alpha=1.02$, i.e., $\bar{L}=288 \mathrm{~m}$. The maximum angle $\theta_{\max }$ which guarantees $L>L_{\min }\left(\theta_{\max }\right)$ is $\theta_{\max }=80.3 \mathrm{deg}$.

Figure 17 shows the chain angle (a), CS velocity magnitude (b) and CS mass (c) as a function of time, during the angular acceleration of the siphon using the sequence described above, assuming $n=40, M(0)=50$ and $D=0$. The braking phases are plotted with a red line. It takes approximately $175 \mathrm{~h}$ to reach the maximum amplitude $\theta_{\max }$ and the CS mass at this point is $M_{f}=$ 1937. In such conditions, the maximum velocity reached by the CS at $\theta=0$ is $\bar{v}_{c s}^{\max }=12.2 \mathrm{~cm} \mathrm{~s}^{-1}$. Note that variations of the initial CS mass (that depends on the volume of material the CS can host, the mass of the processing units and other sub-systems) will clearly affect $M_{f}$ and the time required to reach $\theta_{\max }$. In particular, larger $M(0)$ will lead to larger final mass and longer time to reach the maximum amplitude.

Once $\theta_{\max }$ is reached two different methods are proposed to deliver material to one of the two stable CEP. In the first scenario, the entire CS is released from the siphon and inserted into the PO intersecting the CEPs, whereas in the second scenario the CS remains attached to the chain 


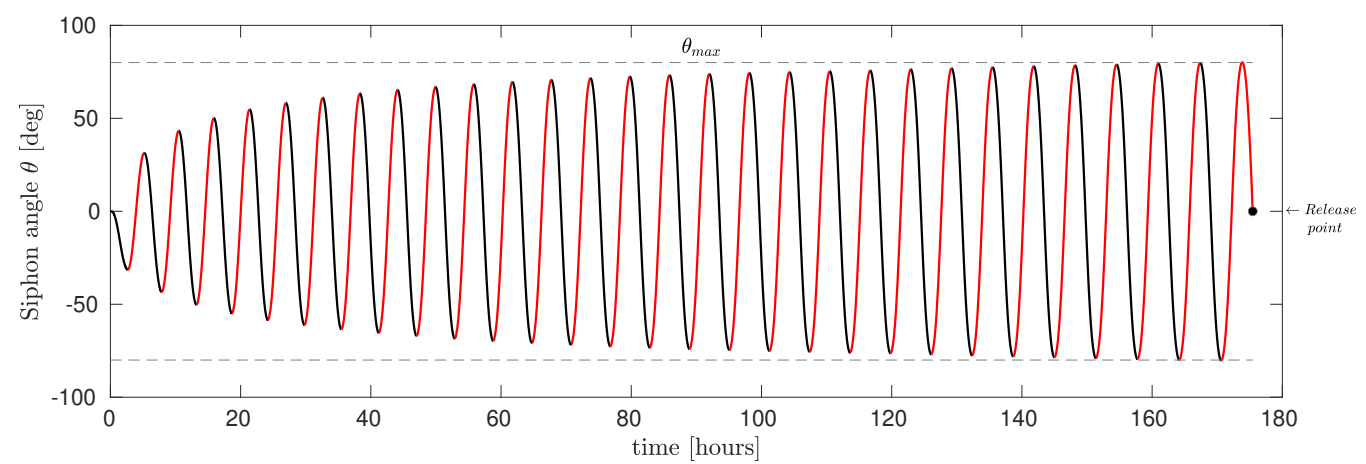

(a)

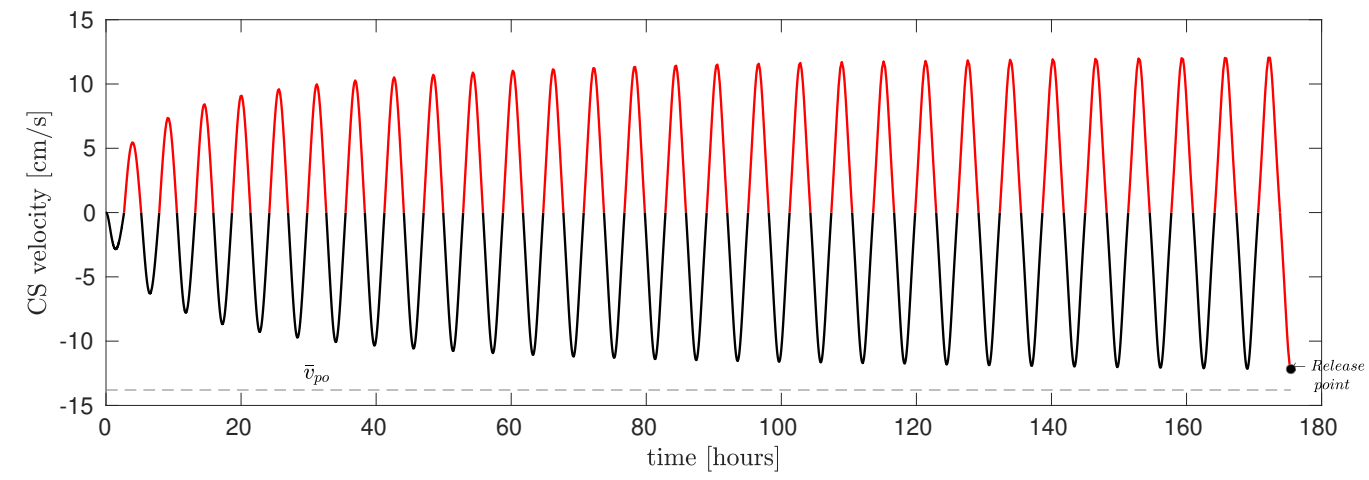

(b)

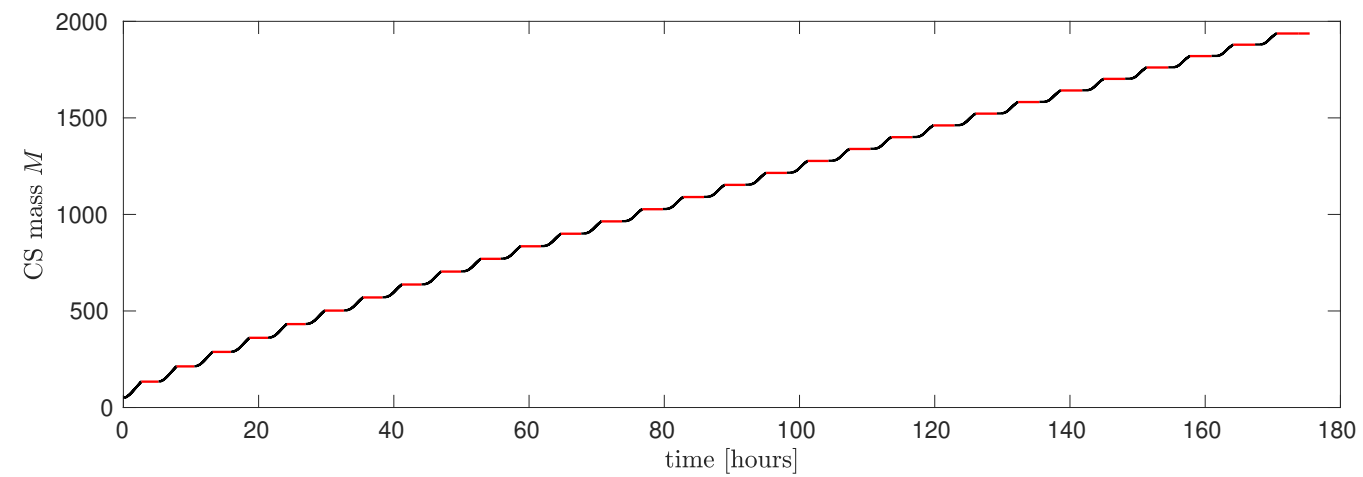

(c)

Figure 17: Chain angle (a), CS velocity (b) and CS mass (c) as a function of time during the acceleration process. The red lines represent the braking phases, when $\dot{s}=0$. 
(a)

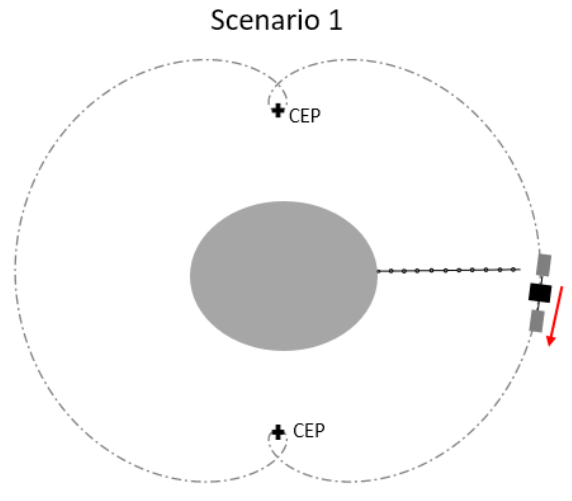

(b)

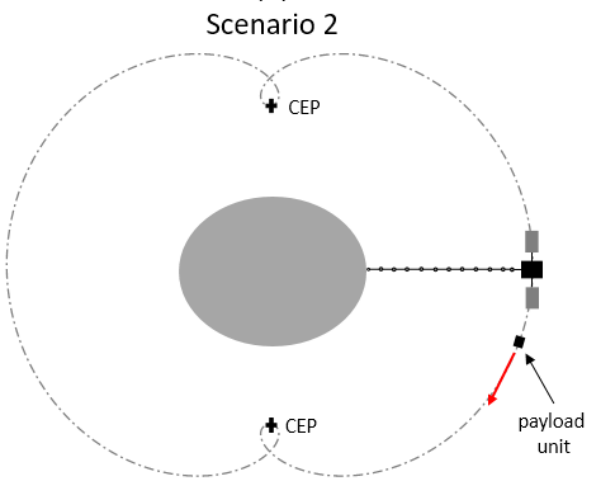

Figure 18: Two proposed release scenarios to transfer payload material from the siphon to the CEP. (a): the entire CS is released from the siphon once the maximum CS velocity $v_{c s}^{\max }$ is reached. (b): a payload unit is released from the CS, while the CS remains attached to the siphon.

and sub-units of payload material are inserted into the PO intersecting the CEPs (Fig. 18).

Scenario 1. The entire CS is released from the siphon and inserted into the PO by applying a small $\Delta \bar{v}=\bar{v}_{p o}-\bar{v}_{c s}^{\max }=1.6 \mathrm{cms}^{-1}$. The CS will then release the material to a catcher located at one of the two CEPs while the siphon oscillation is reduced through a dissipation mechanism at the anchor. After one period of the PO, the CS will dock with the siphon, transferring its angular momentum and thus inducing a rotation on the siphon. Assuming a perfectly inelastic impact, the siphon angular velocity $\dot{\theta}$ after docking will be

$$
\dot{\theta}=\frac{M_{0} L v_{p o}}{\sum_{i=1}^{2 n} m_{i} s_{i}^{2}+M_{0} L^{2}}
$$

with $M_{0}=M(0)$. The delivery of payloads to the CS then restarts, until $M_{f}$ is reached again and the process continues. Note that, due to $\dot{\theta} \neq 0$ after the CS docking, the time to reach $\theta_{\max }$ will be shorter in this phase.

The overall outcome of this process is the periodic transfer of material from the asteroid surface to a $\mathrm{CEP}$ with $\Delta \bar{v}<\Delta \bar{v}_{\text {direct }}^{\min }$.

Scenario 2. In this scenario, the material collected is organized into sub-units with mass $\delta M$, then accelerated by the CS through a spring system or a linear actuator to reach $v_{p o}$. When a single unit 
$\delta M$ is released, the siphon angular velocity decreases to conserve the overall angular momentum:

$$
\left(\sum_{i=1}^{2 n} m_{i} s_{i}^{2}+M L^{2}\right) \dot{\theta}_{1}=\left(\sum_{i=1}^{2 n} m_{i} s_{i}^{2}+(M-\delta M) L^{2}\right) \dot{\theta}_{2}+L \delta M v_{p o}
$$

where $\dot{\theta}_{1}$ and $\dot{\theta}_{2}$ are the siphon angular velocities before and after the $\Delta v$. If $\delta M<<M$ and the inertia of the chain is much smaller than the inertia of the CS (which is true at the end of the acceleration process) then

$$
\dot{\theta}_{2} \approx \dot{\theta}_{1}\left[1-\frac{\delta M}{M} \frac{\Delta v / L}{\dot{\theta}_{1}}\right]
$$

If the chain is stopped while the masses $\delta M$ are inserted into the $\mathrm{PO}$, the angular velocity of the chain would decrease at each unit release according to Eq. (40) (since the CS mass $M$ would decrease). This problem can be circumvented by continuously delivering mass to the CS (using the same sequence described at the beginning of this section) while releasing units $\delta M$ after each period of oscillation of the siphon. Nonetheless, since it is not possible to tune $\delta M$ such that the rate of incoming/outcoming mass to/from the CS is the same, part of the material delivered to the CS has to be sent to escape, in order to keep the CS mass constant.

Using Eq. (39) coupled with the siphon equations of motion (20) it can be shown that $\delta M=1.65$ units of mass can be transferred to the CEP every $6.4 \mathrm{~h}$ at the cost of 56 units of mass released to escape in the same amount of time, while keeping $\Delta \bar{v}<\Delta \bar{v}_{\text {direct }}^{\text {min }}$.

The large ratio between the escape mass and the released mass in the second scenario makes such solution less viable than the first one. However, a combination between the two methods can be used. Whenever a large fraction of material has to be delivered to the CEPs for storing, the method described in Scenario 1 is used. Conversely, if a large fraction of material has to be released to escape (e.g., waste) the double release sequence described in Scenario 2 is used. Although both methods pose a number of undoubtedly challenging engineering issues that should be further discussed and the effects of additional perturbations (e.g., solar radiation pressure) should be included in the future models, this preliminary study has shown that the siphon dynamics can be leveraged to deliver resource payloads to the stable equilibrium points with smaller $\Delta V$ than direct transfer from the asteroid surface using mass drivers, which can be beneficial in a large scale mining scenario. 


\subsection{Transfers to retrograde orbits}

It was noted in Sect 2.1 that fast rotators are characterised by unstable CEPs. In this case, material transfer to CEPs would not be effective, since small perturbations, for example due to solar radiation pressure, will cause the material to migrate from the equilibrium towards the asteroid or to escape. Moreover, when considering more complex asteroid shape models, the stability of the centres might be very sensitive to small variations in the asteroid gravity field and shape [19]. A different strategy for material transfer into orbit can be envisaged if CEPs are unstable, taking advantage of retrograde periodic orbits. Retrograde orbits rotate opposite to the asteroid (with respect to inertial space) and they are generally more stable than direct orbits or EPs [14].

In this case, the orbiting catcher would be placed on a stable retrograde orbit intersecting the CS when $\theta=0$, and material is periodically transferred from the CS to the orbiting catcher, each time the catcher completes one revolution around the asteroid (or at multiples of the revolution period).

For simplicity, an example referred to retrograde orbits around Bennu is proposed here, however this method can be easily extended to other asteroids with unstable CEPs. Figure 19a shows a family of retrograde periodic orbits around Bennu. A lower bound on the minimum orbit altitude can be defined to ensure that a siphon with $L>L_{\min }$ can reach the orbit. In principle any stable orbit in the family above such minimum altitude can then be selected as the nominal catcher orbit, even though higher orbits will have a larger relative velocity with respect to the asteroid, thus increasing the required energy for transfer to the catcher. Take, for example, the red orbit in Fig. 19a that intersects the $x$-axis in the same point as the direct orbit used in the previous section, for transfers to CEPs. Figure $19 \mathrm{~b}$ shows the velocity of the red periodic orbit over one period. At the intersection with the $x$-axis, the velocity is about $34 \mathrm{~cm} \mathrm{~s}^{-1}$, more than two times larger than the velocity of the direct orbit in Fig. 16a passing through the same point. However, using the same swing-and-release technique described in the previous section, an additional $\Delta v \approx 21 \mathrm{~cm} \mathrm{~s}^{-1}$ is required to match with the velocity of the catcher at the intersection with the $x$-axis. In comparison, the minimum $\Delta v$ for insertion into the same periodic orbit from the asteroid surface (calculated using Eq. (37) ) is approximately $32 \mathrm{~cm} \mathrm{~s}^{-1}$, suggesting that, even in this case, the siphon might reduce the overall $\Delta v$ requirements in a large scale mining scenario. 


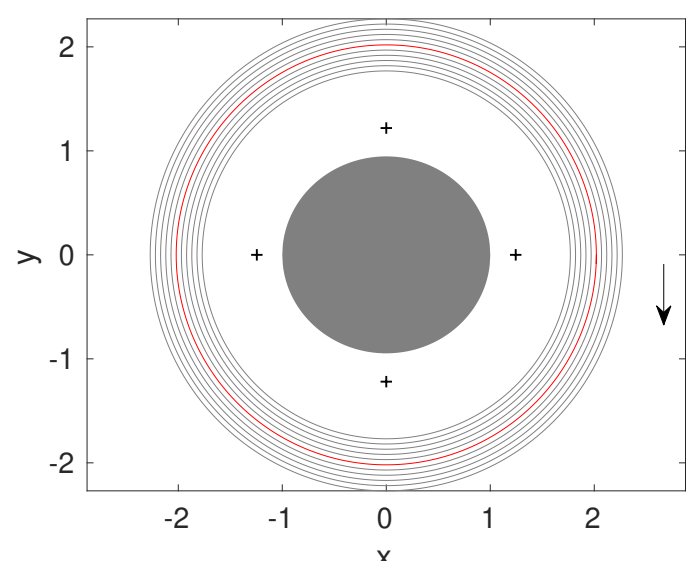

(a)

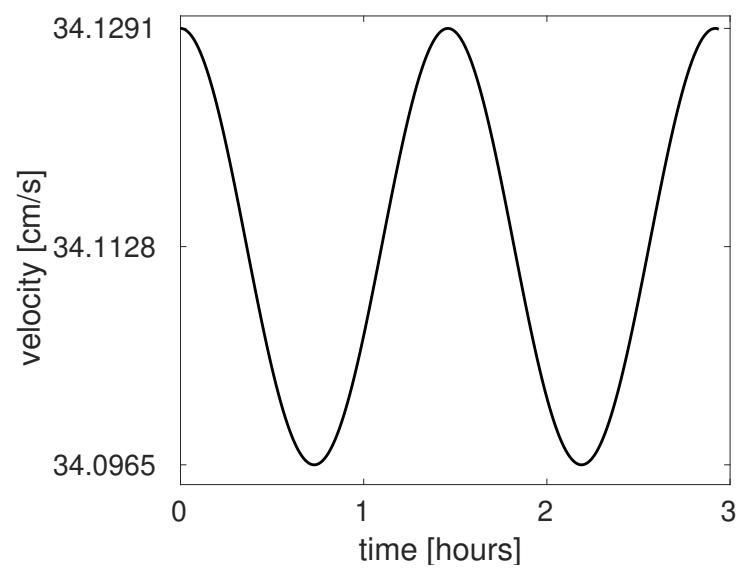

(b)

Figure 19: (a) Stable periodic retrograde orbits around Bennu. (b) Velocity profile for the periodic orbit displayed in red. The largest velocity is achieved at the intersection with the $x$ axis.

\section{Conclusions}

In this paper the behaviour of an orbital siphon anchored to an ellipsoidal asteroid has been investigated. The siphon is envisaged as a chain of tethered-connected bucket masses containing asteroid material (the payload). The buckets are free to slide frictionless on a support structure anchored at the asteroid. If the chain is long enough, the centrifugal-induced forces on the uppermost buckets can be large enough to pull the lower masses, thus initializing an orbital siphon effect: the top payload masses are released while new payloads are connected to the bottom of the chain. In contrast to previous publications on this topic, here large oscillations of the support structure with respect to the anchor are considered and a collecting spacecraft at the top of the siphon is envisaged to collect the payload material released by the siphon.

It has been shown that the siphon effect induces a torque on the chain with respect to the anchor, thus inducing oscillations of the siphon with period comparable to the asteroid period. As more mass is delivered to the collecting spacecraft the amplitude of these oscillations is reduced over time and the velocity of the chain of masses does not diverge but rather reaches a constant value. Longer chains are associated with larger chain velocity and, therefore, a larger rate of material delivered to the CS.

It has been observed that prolate asteroids require a larger minimum chain length if the siphon is anchored at the shortest end. Thus, for a prolate asteroid, the best anchor location of the siphon 
(a)

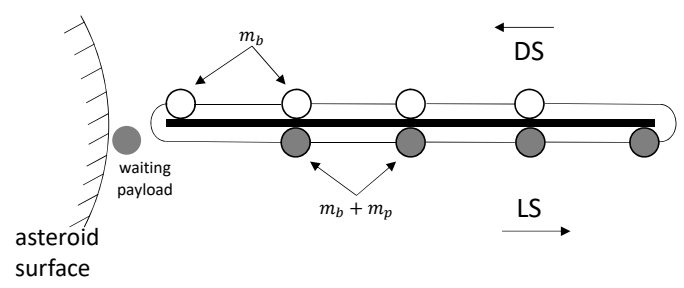

(b)

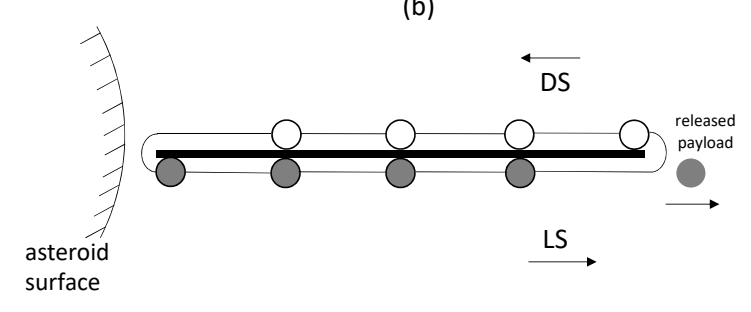

Figure A.1: Chain with $n=4$ before (a) and after (b) the surface bucket is refilled with a payload.

is the longest equatorial end, where the centrifugal-induced acceleration at the surface is larger.

Moreover, it has been shown that the siphon dynamics can be exploited to insert the payloads into a periodic orbit intersecting the centre equilibrium points associated with the effective potential. If these points are stable, they could serve as gravitational depots for material, waiting to be further processed or stored for later collection while the siphon keeps raising resources from the surface of the asteroid.

\section{Appendix A. Modelling payload refilling}

In this section the radial velocity of the chain after the top payload is released and a new one is connected at the surface bucket is calculated, modelling the refilling event as an inelastic collision.

Figure A.1 shows the chain configuration before (a) and after (b) the refilling event, for a chain with $n=4$. The absolute velocity of each mass on the siphon before (superscript I) and after (superscript II) the refilling event is:

$$
\begin{aligned}
\overline{\mathbf{v}}_{i}^{\mathrm{I}} & =\dot{s}^{\mathrm{I}} \hat{\mathbf{u}}_{r}+\overline{\boldsymbol{\omega}} \times \overline{\mathbf{r}}_{i} \\
\overline{\mathbf{v}}_{i}^{\mathrm{II}} & =\dot{s}^{\mathrm{II}} \hat{\mathbf{u}}_{r}+\overline{\boldsymbol{\omega}} \times \overline{\mathbf{r}}_{i}
\end{aligned}
$$

where $\mathbf{r}_{i}$ is the distance between the $i$-th mass and the centre of the asteroid. Similarly, the absolute velocity of the waiting payload (wp) and the released payload (rp) are

$$
\begin{aligned}
& \overline{\mathbf{v}}_{w p}=\overline{\boldsymbol{\omega}} \times \overline{\mathbf{r}}_{w p} \\
& \overline{\mathbf{v}}_{r p}=\dot{s}^{\mathrm{I}} \hat{\mathbf{u}}_{r}+\overline{\boldsymbol{\omega}} \times \overline{\mathbf{r}}_{r p}
\end{aligned}
$$

where it is assumed that the waiting payload is at rest on the asteroid surface. Then, conservation 
of linear momentum dictates:

$$
\bar{m}_{w p} \overline{\mathbf{v}}_{w p}+\sum_{i=1}^{2 n} \bar{m}_{i} \overline{\mathbf{v}}_{i}^{\mathrm{I}}=\bar{m}_{r p} \overline{\mathbf{v}}_{r p}+\sum_{i=1}^{2 n} \bar{m}_{i} \overline{\mathbf{v}}_{i}^{\mathrm{II}}
$$

where the masses of the waiting payload and released payload are the same $\bar{m}_{w p}=\bar{m}_{r p}=\bar{m}_{p}$, whereas $\bar{m}_{i}=\bar{m}_{b}+\bar{m}_{p}$ for a mass on the LS and $\bar{m}_{i}=\bar{m}_{b}$. Substituting Eqs. A.1a and A.2a into Eq. A.3, performing a dot product with $\hat{\mathbf{u}}_{r}$ on both sides and using non-dimensional variables yields:

$$
n \dot{s}^{\mathrm{I}}+D n \dot{s}^{\mathrm{I}}=n \dot{s}^{\mathrm{II}}+(1-D) \dot{s} \mathrm{I}+n D \dot{s}^{\mathrm{II}}
$$

Then, further simplifying:

$$
\dot{s}^{\mathrm{II}}=\frac{n-1}{n} \dot{s}^{\mathrm{I}}
$$

Note that the result is independent of the mass ratio $D$.

\section{Acknowledgements}

CM acknowledges support from a Royal Academy of Engineering Chair in Engineering Technology and a Royal Society Wolfson Research Merit Award.

\section{References}

[1] M. Elvis, Let's mine asteroids for science and profit, Nature 485 (7400) (2012) 549-549. doi: $10.1038 / 485549 \mathrm{a}$.

[2] I. Crawford, M. Elvis, J. Carpenter, Using extraterrestrial resources for science, Astronomy \& Geophysics 57 (4) (2016) 4-32.

[3] C. L. Gerlach, Profitably exploiting near-earth object resources, Proceedings of the 2005 International Space Development Conference, National Space Society, Washington DC.

[4] S. Dorrington, J. Olsen, A location-routing problem for the design of an asteroid mining supply chain network|doi:10.1016/j.actaastro.2018.08.040

URL http://www.sciencedirect.com/science/article/pii/S0094576517306148 
[5] J. McMahon, S. K. Mitchell, K. Oguri, N. Kellaris, D. Kuettel, C. Keplinger, B. Bercovici, Area-of-effect softbots (AoES) for asteroid proximity operations, IEEEdoi:10.1109/aero. 2019.8741680 .

[6] D. G. Yárnoz, J. P. S. Cuartielles, C. R. McInnes, Passive sorting of asteroid material using solar radiation pressure, Journal of Guidance, Control, and Dynamics 37 (4) (2014) 1223-1235. doi:10.2514/1.62412

[7] A. Viale, C. McInnes, M. Ceriotti, Analytical mechanics of asteroid disassembly using the orbital siphon effect, Proceedings of the Royal Society A: Mathematical, Physical and Engineering Sciences 474 (2220) (2018) 20180594. doi:10.1098/rspa.2018.0594.

[8] K. Tsiolkovsky, Dreams of Earth and Sky, The Minerva Group, Inc.

[9] Y. Artsutanov, V kosmos na elektrovoze, Komsomolskaya Pravda 31 (1960) 946-947.

[10] C. R. McInnes, C. Davis, Novel payload dynamics on space elevator systems, 56th International Astronautical Congress, IAC-05-D4.2-07doi:10.2514/6.iac-05-d4.2.07.

[11] A. Viale, C. R. McInnes, M. Ceriotti, Disassembly of near earth asteroids by leveraging rotational self energy, 69th International Astronautical Congress, IAC-18-D4.3-18. URL http://eprints.gla.ac.uk/168724/

[12] A. Viale, C. McInnes, M. Ceriotti, Dynamics of a non-rigid orbital siphon at a near-earth asteroid, Journal of Guidance, Control, and Dynamics (accepted for publication).

[13] D. H. Andrews, The theory of the potential (MacMillan, william duncan), Journal of Chemical Education 7 (10) (1930) 2530. doi:10.1021/ed007p2530.

URL https://doi.org/10.1021\%2Fed007p2530

[14] D. J. Scheeres, Dynamics about uniformly rotating triaxial ellipsoids: Applications to asteroids 110 (2) 225-238. doi:10.1006/icar.1994.1118

URL http://www.sciencedirect.com/science/article/pii/S0019103584711183

[15] W. Szyszkowski, D. Stilling, On damping properties of a frictionless physical pendulum with a moving mass, International Journal of Non-Linear Mechanics 40 (5) (2005) 669-681. doi: 
10.1016/j.ijnonlinmec.2004.09.001.

URL https://doi.org/10.1016\%2Fj .ijnonlinmec.2004.09.001

[16] D. Scheeres, C. Hartzell, P. Sánchez, M. Swift, Scaling forces to asteroid surfaces: The role of cohesion, Icarus 210 (2) (2010) 968-984. doi:10.1016/j.icarus.2010.07.009.

[17] D. Scheeres, S. Hesar, S. Tardivel, M. Hirabayashi, D. Farnocchia, J. McMahon, S. Chesley, O. Barnouin, R. Binzel, W. Bottke, M. Daly, J. Emery, C. Hergenrother, D. Lauretta, J. Marshall, P. Michel, M. Nolan, K. Walsh, The geophysical environment of bennu, Icarus 276 (2016) 116-140. doi:10.1016/j.icarus.2016.04.013.

URL https://doi.org/10.1016\%2Fj .icarus.2016.04.013

[18] K. C. Howell, Three-dimensional, periodic, halo orbits, Celestial Mechanics 32 (1) (1984) 5371. doi:10.1007/bf01358403.

URL https://doi.org/10.1007\%2Fbf01358403

[19] D. J. Scheeres, , J. W. McMahon, A. S. French, D. N. Brack, S. R. Chesley, D. Farnocchia, Y. Takahashi, J. M. Leonard, J. Geeraert, B. Page, P. Antreasian, K. Getzandanner, D. Rowlands, E. M. Mazarico, J. Small, D. E. Highsmith, M. Moreau, J. P. Emery, B. Rozitis, M. Hirabayashi, P. Sánchez, S. V. wal, P. Tricarico, R.-L. Ballouz, C. L. Johnson, M. M. A. Asad, H. C. M. Susorney, O. S. Barnouin, M. G. Daly, J. A. Seabrook, R. W. Gaskell, E. E. Palmer, J. R. Weirich, K. J. Walsh, E. R. Jawin, E. B. Bierhaus, P. Michel, W. F. Bottke, M. C. Nolan, H. C. Connolly, D. S. Lauretta, The dynamic geophysical environment of (101955) bennu based on OSIRIS-REx measurements, Nature Astronomy 3 (4) (2019) 352-361. doi:10.1038/s41550-019-0721-3

URL https://doi.org/10.1038\%2Fs41550-019-0721-3 